\title{
How to maximise the value of residual biomass resources: The case of straw in Denmark
}

Venturini, Giada; Pizarro Alonso, Amalia Rosa; Münster, Marie

Published in:

Applied Energy

Link to article, DOI:

10.1016/j.apenergy.2019.04.166

Publication date:

2019

Document Version

Peer reviewed version

Link back to DTU Orbit

Citation (APA):

Venturini, G., Pizarro Alonso, A. R., \& Münster, M. (2019). How to maximise the value of residual biomass resources: The case of straw in Denmark. Applied Energy, 150, 369-388.

https://doi.org/10.1016/j.apenergy.2019.04.166

\section{General rights}

Copyright and moral rights for the publications made accessible in the public portal are retained by the authors and/or other copyright owners and it is a condition of accessing publications that users recognise and abide by the legal requirements associated with these rights.

- Users may download and print one copy of any publication from the public portal for the purpose of private study or research.

- You may not further distribute the material or use it for any profit-making activity or commercial gain

- You may freely distribute the URL identifying the publication in the public portal

If you believe that this document breaches copyright please contact us providing details, and we will remove access to the work immediately and investigate your claim. 


\title{
How to maximise the value of residual biomass resources: The case of straw in Denmark
}

\author{
Venturini, Giada; Pizarro Alonso, Amalia Rosa; Münster, Marie
}

Published in:

Applied Energy

Publication date:

2021

Document Version

Peer reviewed version

Link back to DTU Orbit

Citation (APA):

Venturini, G., Pizarro Alonso, A. R., \& Münster, M. (Accepted/In press). How to maximise the value of residual biomass resources: The case of straw in Denmark. Applied Energy.

\section{General rights}

Copyright and moral rights for the publications made accessible in the public portal are retained by the authors and/or other copyright owners and it is a condition of accessing publications that users recognise and abide by the legal requirements associated with these rights.

- Users may download and print one copy of any publication from the public portal for the purpose of private study or research.

- You may not further distribute the material or use it for any profit-making activity or commercial gain

- You may freely distribute the URL identifying the publication in the public portal

If you believe that this document breaches copyright please contact us providing details, and we will remove access to the work immediately and investigate your claim 


\title{
How to maximise the value of residual biomass resources: The case of straw in Denmark
}

\author{
Giada Venturini $\varpi^{\mathrm{a}}$, Amalia Pizarro-Alonso ${ }^{\mathrm{a}}$, Marie Münster ${ }^{\mathrm{a}}$ \\ ${ }^{a}$ Department of Management Engineering, Technical University of Denmark, 2800 Kgs. Lyngby, Denmark
}

\begin{abstract}
The long-lived dependence on fossil fuels has led to a slow pace in the transition to renewable energy sources in the heavy-duty sectors of the energy system. While bioenergy might represent a possible alternative, biomass is a limited resource, whose use is restricted by potential technical, environmental and social implications. Because residual biomass inherently minimises these negative impacts, when its sustainable use is ensured, it could lend itself to multiple options, including production of back-up power, heating and alternative transport fuels. This study investigates different pathways for the optimal use of the most abundant residual biomass in Denmark, i.e. straw, from a technical, economic and environmental perspective. We harness the strengths of two bottom-up model typologies by means of soft-linkage to reveal insights from both perspectives: the multi-sectoral model, TIMESDK, provides a system assessment of the whole energy sector, while the geographically detailed optimization model, Balmorel-OptiFlow, supports the analysis of biorefinery plant size, location and area-specific recovery of excess heat. Modelling results of carbon- and resource-constrained energy scenarios reveal the increased value of straw in a future decarbonised energy system and the attractiveness of the gasification route with Fischer-Tropsch synthesis for the production of biofuels to supply the heavy segments of the transport sector. Moreover, relying on current domestic biomass resources would not attain the energy self-sufficiency targets in a carbon-constrained case.
\end{abstract}

Keywords: Energy systems analysis, Optimization, Energy modelling, Straw, Biomass, Biorefineries

\section{Introduction}

The transition to a future low-carbon energy system presents numerous challenges. Biomass and bioenergy might play an important role in future energy systems according to several scenario studies (Guo, Song, and Buhain, 2015), (Junginger, Bolkesjø, Bradley, Dolzan, Faaij, Heinimö, Hektor, Øyvind Leistad, Ling, Perry, Piacente, Rosillo-Calle, Ryckmans, Schouwenberg, Solberg, Trømborg, da Silva Walter, and de Wit, 2008), (Berndes, Hoogwijk, and Van Den Broek, 2003). Nevertheless, researchers warn against the potential negative implications associated with an extensive use of biomass for energy purposes, due to e.g. the impacts on use of land, food and water systems (Robledo-Abad, Althaus, Berndes, Bolwig, Corbera, Creutzig, Garcia-Ulloa, Geddes, Gregg, Haberl, Hanger, Harper, Hunsberger, Larsen, Lauk, Leitner, Lilliestam, Lotze-Campen, Muys, Nordborg, Ölund, Orlowsky, Popp, Portugal-Pereira, Reinhard, Scheiffle, and Smith, 2017), (Parajuli, Dalgaard, Jørgensen, Adamsen, Knudsen, Birkved, Gylling, and Schjoerring, 2015), (Gregg and Smith, 2010).

Biomass resources largely fall into three main categories: natural biomass (i.e. forest), energy crops and residual biomass. Within the latter, Brosowski, Thraen, Mantau, Mahro, Erdmann, Adler, Stinner, Reinhold, Hering, and Blanke (2016) distinguish five high-level groups: agricultural by-products, residues of forestry and wood industries, municipal waste, industrial residues, and residues from other

Email address: venturini.giada@gmail.com (Giada Venturini $₫$ ) 
areas. Common examples are cereal straw, rice husks, corn stover, animal slurry and manure, logging residues, sawdust, and fruit shells. Residual biomass resources are considered by-products if no further value is extracted within the food, farming or wood industry. However, when considering cereal straw, for instance, its uses range from animal bedding, animal feed, mushroom production and frost protection in horticulture, to roof thatching and wall panel production (Spøttle, Alberici, Toop, Peters, Gamba, Ping, van Steen, and Bellefleur, 2003). Moreover, ploughing straw in the field contributes to soil fertility and physical structure (Powlson, Riche, Coleman, Glendining, and Whitmore, 2008).

In Denmark, the main current use for residual biomass from agriculture is "left on field" (Tonini, Hamelin, and Astrup, 2016). Straw from wheat, barley and other cereals amounts to $4900 \mathrm{t}$ DM/year (dry matter/year), corresponding to $68 \mathrm{PJ} /$ year, with around $50 \%$ being left on field, $23 \%$ used for energy, and $27 \%$ for feeding bedding (Statistics Denmark, 2016). Nature grass, thus not including garden waste, is around $430 \mathrm{t} \mathrm{DM} /$ year $(6.7 \mathrm{PJ} /$ year $)$, with all of it currently being left on field (Tonini, Hamelin, and Astrup, 2016). Animal manure corresponds to 6200 t DM/year, with 4\% used for energy and 96\% left on field (Tonini, Hamelin, and Astrup, 2016), while Scarlat, Fahl, Dallemand, Monforti, and Motola (2018) report that the collectable manure amounts to 2930 t DM/year. Given these estimates, residual biomass from agriculture holds an annual energy potential in the range of 105-145 PJ/year, corresponding to 14-19\% of the total primary energy consumption in Denmark in 2015 (Danish Energy Agency, 2017a). A recent analysis by Tonini, Vadenbo, and Astrup (2017) reports similar values for the potential of residual biomass from agriculture. Furthermore, the energy potential from wood residues ranges between 5.6 PJ/year (Astrup, Tonini, Hamelin, and Wenzel, 2011) and 12.6 PJ/year (Danish Energy Agency, 2013), with all woody biomass potential estimated at around $40 \mathrm{PJ} /$ year (Astrup, Tonini, Hamelin, and Wenzel, 2011). Other agro-industrial residues, such as, e.g. beet pulp and molasses, are fully destined to fodder production (Tonini, Hamelin, and Astrup, 2016); hence, they will not be considered hereafter.

Given the potential of this partly untapped resource, an increasing number of studies investigate the utilisation of agricultural residues as raw materials for electricity and heat production (Schmidt, Gass, and Schmid, 2011), (Turconi, Tonini, Nielsen, Simonsen, and Astrup, 2014), (Weiser, Zeller, Reinicke, Wagner, Majer, Vetter, and Thraen, 2014), (Panos and Kannan, 2016), (Bentsen, Nilsson, and Larsen, 2018) or in biorefinery systems (Cherubini and Ulgiati, 2010),(Steubing, Zah, and Ludwig, 2012), (Ekman, Wallberg, Joelsson, and Borjesson, 2013), (Wenzel, Høibye, Duban Grandal, Hamelin, Bird, and Olesen, 2014), (Pettersson, Wetterlund, Athanassiadis, Lundmark, Ehn, Lundgren, and Berglin, 2015), (Tonini, Hamelin, and Astrup, 2016), (Parajuli, Kristensen, Knudsen, Mogensen, Corona, Birkved, Peña, Graversgaard, and Dalgaard, 2017), (Tonini, Vadenbo, and Astrup, 2017). Technological options considered in the aforementioned studies comprise bioethanol production via fermentation, biogas from anaerobic co-digestion with manure, synthetic fuels from thermal gasification, and combustion for heat and power generation.

In particular, in the Danish context, researchers approached the use of residual biomass for energy and fuel production from a number of perspectives, often yielding discordant results on which pathway to prioritise. Several works applied comprehensive Life Cycle Assessments to estimate the environmental consequences associated with the use of residual biomass (Tonini, Vadenbo, and Astrup, 2017), (Parajuli, Kristensen, Knudsen, Mogensen, Corona, Birkved, Peña, Graversgaard, and Dalgaard, 2017), (Wenzel, Høibye, Duban Grandal, Hamelin, Bird, and Olesen, 2014), (Turconi, Tonini, Nielsen, Simonsen, and Astrup, 2014), (Nguyen, Hermansen, and Mogensen, 2013), while others focused on the impact on the use of land (Larsen, Jepsen, and Frederiksen, 2013), (Gylling, Jørgensen, Bentsen, Kristensen, Dalgaard, Felby, Larsen, and Johannsen, 2016) and soil (Hamelin, Naroznova, and Wenzel, 2014). Some studies examined the issue from a techno-economic perspective (Sigurjonsson, Elmegaard, Clausen, and Ahrenfeldt, 2015), (Connolly, Mathiesen, and Ridjan, 2014), (Moller, Slento, and Frederiksen, 2014) and other works included considerations on the socioeconomic effects, e.g. job creation (Gylling, Jørgensen, Bentsen, Kristensen, Dalgaard, Felby, Larsen, and Johannsen, 2016), (Moller, Slento, and Frederiksen, 2014). Moreover, other studies have analysed the recycling of nutrients and carbon into the soil from the products of biomass gasification (Sigurjonsson, Elmegaard, Clausen, and Ahrenfeldt, 2015) and anaerobic digestion (Hamelin, Naroznova, 
and Wenzel, 2014), which can bring about positive effects for the energy system and the environment. Also, the generation of non-energy co-products, such as feed, contributes to a circular use of resources (Tonini, Vadenbo, and Astrup, 2017).

For the case of straw, multiple alternative uses have been identified, each one with its advantages and limitations. The fermentation pathway for the production of second-generation bioethanol yields valuable co-products, such as animal fodder and lignin, the latter re-usable for power and heat generation. Although the technology could provide sustainable fuels for the light transport sector, no commercial bioethanol production from ligno-cellulosic material exists, with optimization of enzymes use, straw pre-treatment and process yield being the main challenges (Danish Energy Agency, 2016), (Larsen, Haven, and Thirup, 2012). Co-digestion of straw with manure and other feedstocks for the production of biogas represents a competitive use, with the possibility of recycling the digestate to restore soil carbon and nutrients (Tonini, Hamelin, and Astrup, 2016), (Hamelin, Naroznova, and Wenzel, 2014). On the other hand, due to the high lignin content in straw, degradation time increases considerably and pre-treatment is needed to ensure adequate biogas yields at lower retention times. Fischer-Tropsch (FT) synthesis of syngas from straw gasification could yield a variety of synthetic fuels, e.g. methanol or DME (dimethyl ether) (Connolly, Mathiesen, and Ridjan, 2014), which can replace fossil fuel combustion in vehicles with minor engine modifications. The flexibility in the output fuels, and the option of storing surplus electricity into electrofuels, from carbon dioxide and water electrolysis (via hydrogen), thus boosting the production of liquid or gaseous fuels, represent the main advantages of this technology, while process efficiency improvements, fuel handling and infrastructure costs remain more uncertain parameters (Brynolf, Taljegard, Grahn, and Hansson, 2018).

While a number of studies are attempting to integrate the environmental assessment of these options within energy system analysis, in Denmark (Tonini, Vadenbo, and Astrup, 2017) and globally (Samsatli, Samsatli, and Shah, 2015), often relevant techno-economic considerations remain out of the study scope. For instance, a detailed spatial and temporal resolution is required for capturing biomass cycles, the site of its generation and the place of its use, which in turn affect, e.g. storage and transport costs. Furthermore, the location and size of conversion plants, for power, heat and fuel production, could be optimised while considering the trade-offs between economies of scale and transportation of biomass resources (Shu, Schneider, and Scheffran, 2017), (Wetterlund, Leduc, Dotzauer, and Kindermann, 2012). Sharma, Ingalls, Jones, and Khanchi (2013) and Yue, You, and Snyder (2014) offer a review of studies addressing design and optimization of biomass supply chains. Both simulation (Daioglou, Wicke, Faaij, and Van Vuuren, 2015) and optimization models (Forsell, Guerassimoff, Athanassiadis, Thivolle-Casat, Lorne, Millet, and Assoumou, 2013), (Samsatli, Samsatli, and Shah, 2015), (Hagberg, Pettersson, and Ahlgren, 2016) are adopted for incorporating the biomass supply chain in energy system models. Integrated modelling and model linking combine the strengths of different models, e.g. energy system models and land use models (Gonzalez-Salazar, Venturini, Poganietz, Finkenrath, Kirsten, Acevedo, and Spina, 2016), (van Dyken, Bakken, and Skjelbred, 2010), (Schmidt, Gass, and Schmid, 2011). Moreover, spatially disaggregated tools allow for a detailed representation of cost-supply curves (Forsell, Guerassimoff, Athanassiadis, ThivolleCasat, Lorne, Millet, and Assoumou, 2013), technology pathways (Samsatli, Samsatli, and Shah, 2015) and industry integration (Pettersson, Wetterlund, Athanassiadis, Lundmark, Ehn, Lundgren, and Berglin, 2015).

The present study investigates different pathways for the optimal use of the most abundant residual biomass resource in Denmark, i.e. straw, applying both techno-economic constraints and climate goals. We harness the strengths of two model typologies linked to reveal insights from two perspectives: the multi-sectoral model TIMES-DK provides a system assessment of the whole energy sector, including the representation of energy technologies and their competition in supplying the electricity, heat and transport demands; on the other hand, the geographically and temporally detailed optimization model Balmorel, with the add-on OptiFlow, supports the analysis of biomass transport costs and area-specific recovery of heat for district heating $(\mathrm{DH})$ networks. Stemming from a common cost-optimization framework, model linking allows capturing many dimensions, including inter-sectoral technology competition and environmental considerations associated with biomass use, 
such as indirect land-use changes, changes in soil carbon and fertilising effects on fields.

This paper is structured as follows: Section 2 illustrates the adopted methodology and modelling framework while Section 3 reports the main data assumptions; Section 4 describes the scenarios definition, Section 5 presents the results of our case study, and Section 6 discusses findings, trade-offs and policy implications; finally, Section 7 draws the conclusions.

\section{Methodology}

The generation of residual biomass, such as straw, varies on a regional basis and its utilisation depends on competitive uses, including the trade-offs between transport distances, economies of scales and high-value by-products, such as excess heat from biorefineries. In addition, future energy systems will be increasingly characterised by intermittent renewable energy (RE) sources. This calls for a novel and integrated approach in the field of energy modelling and optimization, comprising: 1) Representation of the whole integrated energy system; 2) Detailed spatial granularity and high temporal resolution with chronology; 3) Integration of environmental considerations associated to the sustainable use of biomass. A single model that could capture all of the above would require large computational times. Therefore, this study proposes a soft-linkage between the model TIMESDK (Section 2.1) and the model Balmorel with the add-on OptiFlow (Section 2.2). To perform a comprehensive analysis of the optimal use of straw, we consider the impact of detailed geographical and temporal dimensions, as well as introduce environmental accounting related to biogenic carbon emissions and soil fertilising effects (Section 2.4).

\subsection{The TIMES-DK model}

The model generator TIMES (The Integrated MARKAL-EFOM System) was developed and is maintained by the Energy Technology Systems Analysis Programme (ETSAP), an Implementing Agreement of the International Energy Agency (IEA), established in 1976.

TIMES is a technology-rich, bottom-up model generator utilised for long-term analysis and planning of regional, national and multi-national energy systems. Furthermore, the techno-economic partial equilibrium model generator assumes full foresight and perfectly competitive markets (Balyk, Andersen, Dockweiler, Gargiulo, Karlsson, Næraa, Petrović, Tattini, Termansen, and Venturini, 2019). TIMES is mathematically formulated as a linear program, minimising the total system cost discounted to the reference year and calculated as sum of investment costs, fixed and variable operation and maintenance costs, import costs, and export revenues for all the processes included.

In particular, TIMES-DK provides a system assessment of the whole energy sector for Denmark, geographically aggregated in two regions, i.e. Denmark-East (DKE) and Denmark-West (DKW), with technological and economic projections until 2050, including assumptions on exogenous prices, end-use demands and resource availability (Balyk, Andersen, Dockweiler, Gargiulo, Karlsson, Næraa, Petrović, Tattini, Termansen, and Venturini, 2019). TIMES-DK includes the representation of energy technologies and their competition in supplying the electricity, heat and transport end-use demands (Balyk, Andersen, Dockweiler, Gargiulo, Karlsson, Næraa, Petrović, Tattini, Termansen, and Venturini, 2019). Endogenous technology learning is currently not included in the model, with projections on technology developments (e.g. costs, efficiencies) provided exogenously as inputs to TIMES-DK.

The inclusion of the most important end-use sectors of the economy, i.e. industry, residential and transport (Figure 1), allows examining the interplay between supply-side and end-use sectors from a system perspective. Since commodities such as fuels, electricity and heat are shared resources, the integrated modelling of these can clarify the existing synergies and competition across sectors. Similarly, the imposition of a climate or energy policy target can illustrate the burden shifting across sectors along the modelling horizon in a techno-economic perspective.

Moreover, fuel supply chains, i.e. fossil, biofuels and electricity, are modelled from production to distribution, along with fuel imports and exports. The fuel infrastructure consists of two components: 


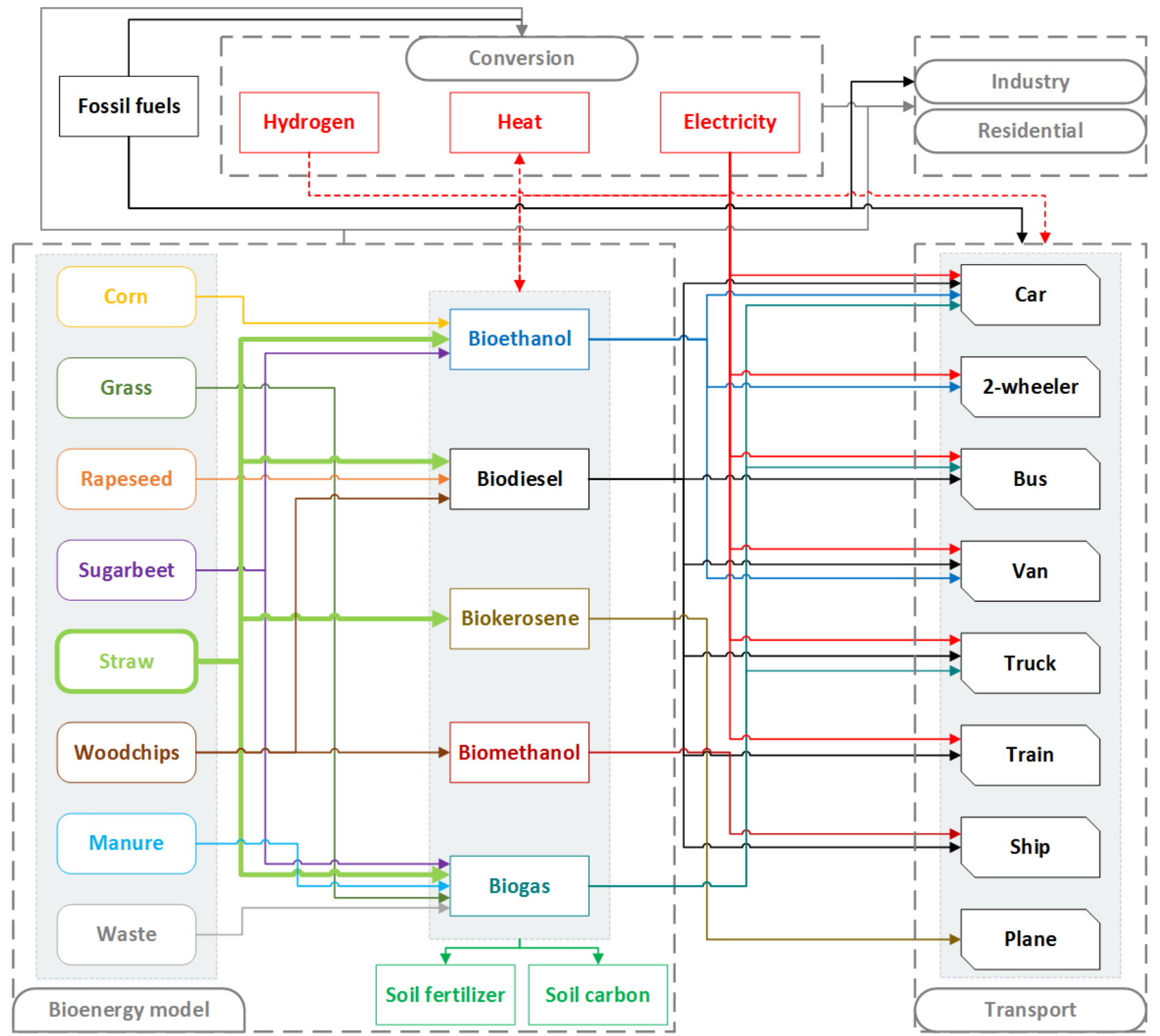

Figure 1: Cross sectoral interactions in TIMES-DK, with focus on bioenergy conversion pathways and fuel options to the end-use sectors. Fossil fuels include natural gas, crude oil and its refined products, i.e. heavy fuel oil, kerosene, gasoline and diesel. Solid biomass types supplying the industry and residential sectors include straw, woodchips, wood pellets and waste, while liquid/gaseous biofuels comprise biodiesel and biogas.

fuel delivery and fuel stations. Fuel delivery from fuel production to the end-use sectors is represented through a variable cost (€/GJ of transported fuel) (Ea Energy Analyses, 2013), (EA Energy Analysis, 2014), assuming that the existing fuel and grid infrastructure can accommodate the annual demand for fuel delivery. Fuel stations are explicitly modelled for public electric vehicle charging and blended gas stations, the latter including both natural gas and biomethane. Virtual technologies simulate power and gas stations, characterised by an investment cost and the information on existing stock in the base year (2015) (International Energy Agency, 2013), (Statistics Denmark, 2018a), (Statistics Denmark, 2018b). In this way, only when the required power/gas for vehicles exceeds the stock, the additional required capacity will be built at a cost equal to the investment cost.

As per the temporal and geographical aggregation, each modelled year is subdivided in 32 nonsequential time slices, built as the combination of factors influencing the variability of $\mathrm{RE}$ sources and end-use energy demands. The factors considered for the definition of the time slices are the four seasons (spring, summer, autumn, winter), two typical days of the week (working, non-working) and four weather-specific cases ("high wind production - low power demand", "high power demand - low wind production", "no photovoltaics (PV) production", "rest"). This time aggregation represents 
the most detailed resolution used in TIMES-DK in this analysis, yet not all modelled parameters are defined at this level of detail, e.g. transport demand is described at annual level. For each of the two regions, DKE and DKW, three areas are defined based on their heat demand: central, decentral and individual. Central and decentral areas are connected to the $\mathrm{DH}$ grid, while individual areas represent suburban or rural zones served by individual heating technologies. For a complete overview of the model TIMES-DK and the mathematical formulation of TIMES model generator, the reader can refer to respectively Balyk, Andersen, Dockweiler, Gargiulo, Karlsson, Næraa, Petrović, Tattini, Termansen, and Venturini (2019) and Loulou, Goldstein, Kanudia, Lettila, and Remme (2016).

\subsection{The Balmorel model with the OptiFlow add-on}

Balmorel is a partial equilibrium model for simultaneous optimization of investments and dispatch of electricity, district heating and hydrogen, including power transmission, under the assumption of perfectly competitive markets (Wiese, Bramstoft, Koduvere, Pizarro Alonso, Balyk, Kirkerud, Tveten, Bolkesjø, Münster, and Ravn, 2018). The reader can find the general mathematical structure of the model in Ravn (2001).

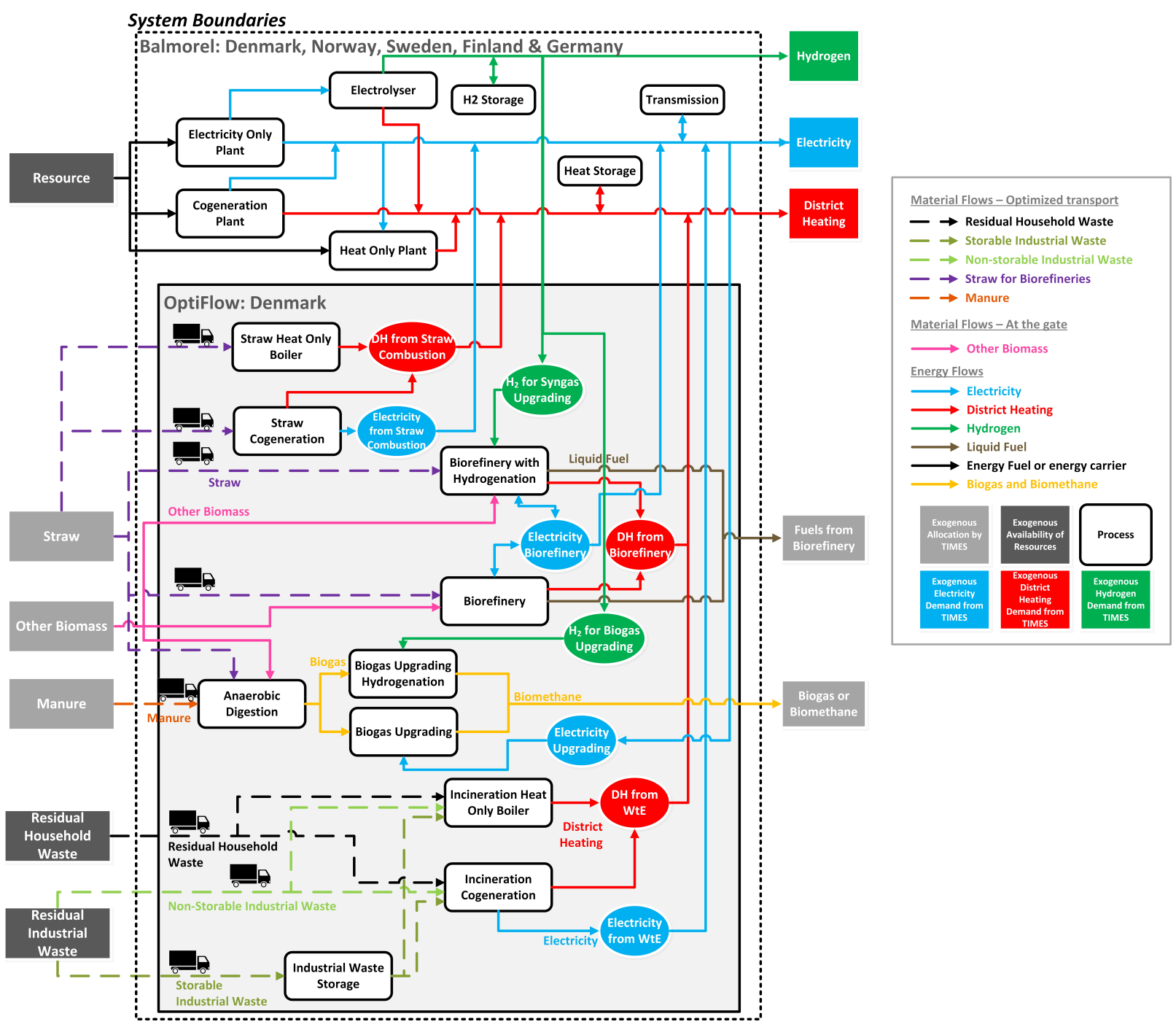

Figure 2: Representation of the energy system, and use of straw, manure and residual waste in Balmorel-OptiFlow (as applied in this study).

The model, exemplified in the upper part of Figure 2, as used in this study, determines the optimal system configuration for satisfying the energy demands by maximising social welfare, i.e. consumers' 
utility minus producers' cost for the electricity, district heating and hydrogen demands, subject to technical, physical and regulatory constraints. The model is data-driven and has a high degree of flexibility with respect to temporal and spatial resolution. For instance, the user-defined temporal dimension allows for different levels of chronological detail, from weekly to hourly levels. For this analysis, an hourly resolution is adopted for four full representative weeks, one for each of the four seasons.

OptiFlow is a generalised network model (Figure 2), whose arcs and nodes topology can be used to analyse the optimal use of residual biomass and waste, integrating economic, environmental and resource scarcity considerations, among others (Pizarro-Alonso, Cimpan, Ljunggren Söderman, Ravn, and Münster, 2018a), (Pizarro-Alonso, Cimpan, and Münster, 2018b). A mathematical introduction to the model is presented in Ravn (2017). OptiFlow is herein used as an integrated add-on within the Balmorel model, i.e. hard-linked, with the former providing the net production of electricity and DH generation from Waste-to-Energy (WtE) and biorefinery plants to the latter, as well as the hydrogen demand for syngas upgrading in biorefineries. In order to model economy of scale endogenously, the model Balmorel-OptiFlow is defined as a mixed integer linear program (MILP).

In this study, district heating and transport of resources are modelled in Balmorel-OptiFlow at a higher geographical resolution than in TIMES-DK. In addition, electricity and district heating generation in Norway, Sweden, Finland and Germany is also modelled, as to represent that the Danish electricity clearing price is determined within the interconnected North European market. For district heating, the 34 largest DH grids in Denmark are represented individually, where the technologies competing for the supply of heat, especially the base-load demand, are waste incineration plants, excess heat from industries and biorefineries. Due to economy of scale, incineration plants and biorefineries can be built only in those 34 larger areas, against the almost 500 medium and small district heating networks existing in Denmark, which are represented in an aggregated way by power regions, i.e. East and West (see blue areas in Figure 3 (a)). To represent the local availability and transportation of biomass and waste resources, another spatial layer is added, with a representation of the 98 municipalities in the country. Transport of resources takes place through the euclidean distances between the centroids of each polygon, i.e. each municipality (as calculated by QGIS 2.14.22), considering only the five closest points for each centroid. Transport of residual resources, i.e. straw and waste, is optimised following the networks depicted in Figure 3 (b). Other biomass types, e.g. energy crops, are assumed to be acquired at the plant gate at their market price, thus the transportation routes and costs are not endogenously modelled.

\subsection{Linkage of TIMES-DK and Balmorel-OptiFlow}

To benefit from the specific strengths of each energy system model, respectively in terms of process coverage and spatio-temporal resolution, TIMES-DK and Balmorel-OptiFlow are soft-linked, as illustrated in Figure 4. The two models are first harmonised in terms of technical and economic characteristics of conversion plants and their stock capacity, exogenous fuel prices, biomass potentials, and renewable energy capacity potentials. Additionally, while TIMES-DK performs a perfect foresight optimization along the whole modelling horizon, Balmorel adopts perfect foresight within the optimization year and a myopic approach between years, as to portray a more realistic picture of technology investments under uncertainty, and to avoid a very high computational time associated with the detailed spatial and temporal resolution adopted.

TIMES-DK calculates annual power, district heating and hydrogen demands as inputs to Balmorel, as well as yearly allocation of residual biomass and fossil fuels for electricity, district heating and fuel production. Subsequently, Balmorel-OptiFlow co-optimises power, district heating and hydrogen generation, along with the utilisation of residual biomass, at a higher geographical and temporal resolution. Subsequently, Balmorel-OptiFlow informs TIMES-DK on the computed average transport cost for straw, the amount of heat that can be efficiently recovered from biorefineries in the DH grids, and the net electricity trade volume between neighbouring power regions. The data is exchanged between the two models until convergence of the fuel mix in the power and district heating sectors. 


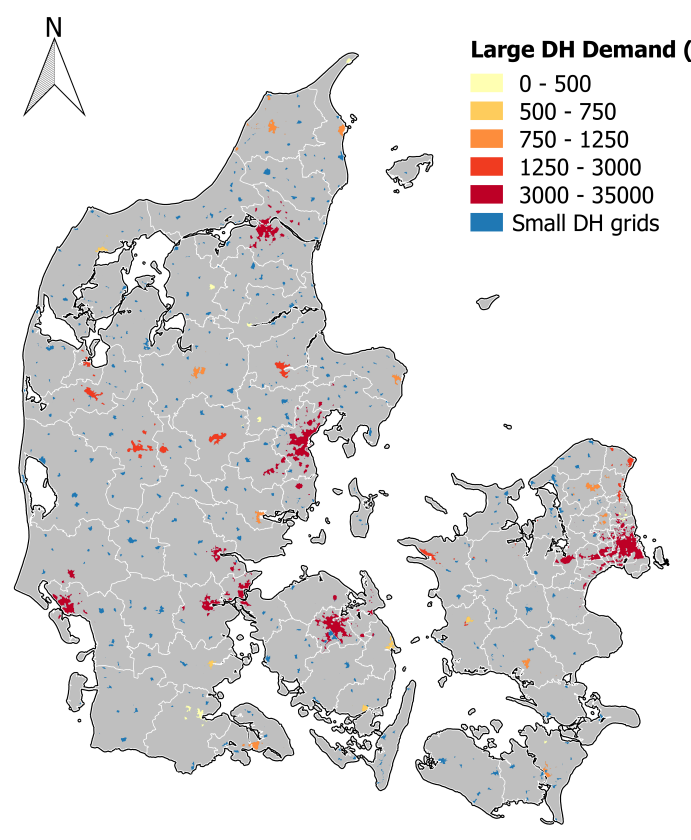

$\begin{array}{llllll}25 & 0 & 25 & 50 & 75 & 100 \\ \mathbf{k m}\end{array}$

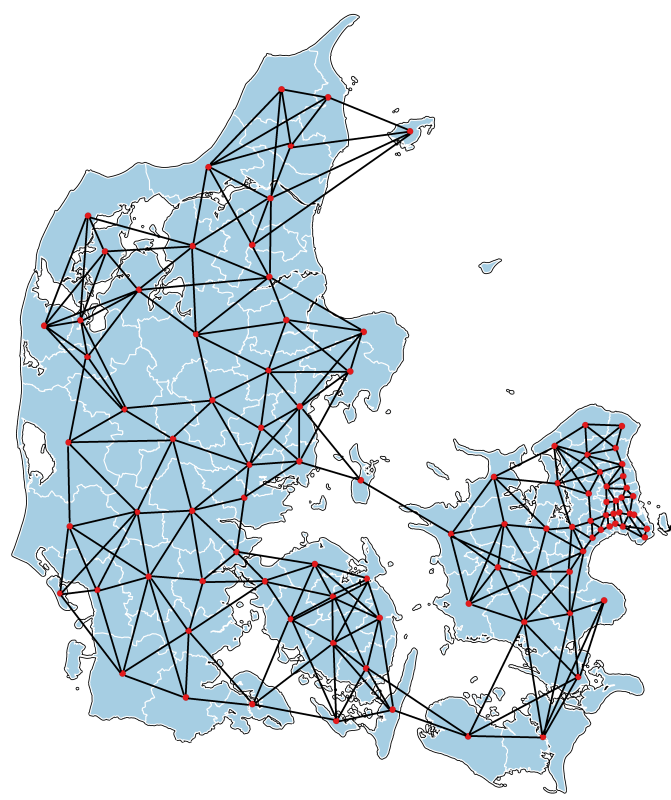

(b)

Figure 3: Geographical representation in Balmorel-OptiFlow: district heating networks in Denmark (a) and geographical areas for biomass collection with transport links (b).

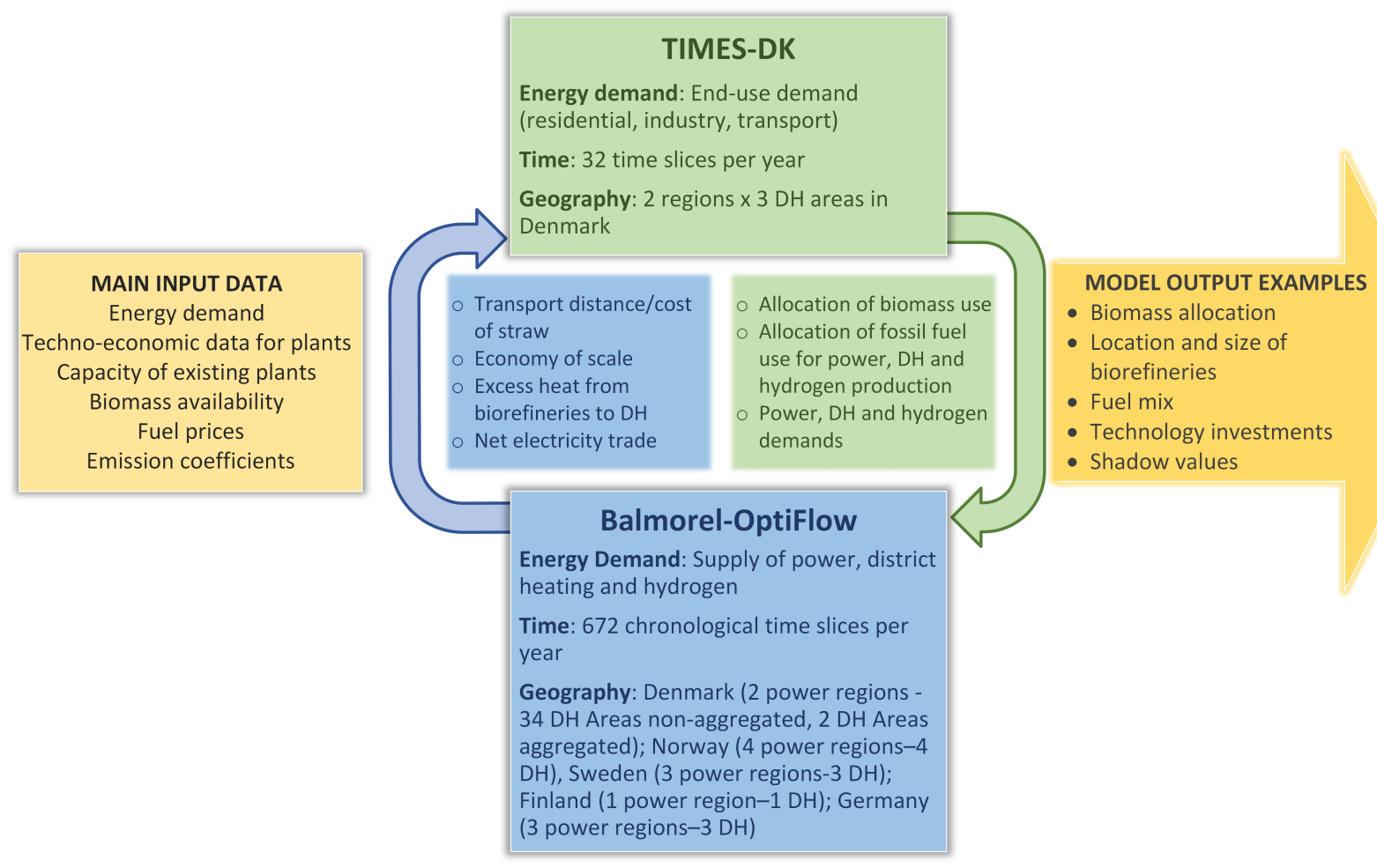

Figure 4: Model linking between TIMES-DK and Balmorel-OptiFlow.

\subsection{Environmental accounting}

In addition to the techno-economic perspective traditionally adopted within energy system analyses, we introduce the representation of two main environmental aspects related to the use of biomass: biogenic carbon accounting and soil fertilising effects.

Both fossil and biogenic $\mathrm{CO}_{2}$ emissions are accounted for, through the assignment of emission factors 
to energy commodities. While fossil emissions contribute to global warming, we assume that direct $\mathrm{CO}_{2}$ emissions from biogenic origin would be neutral in terms of global warming potential, as short biomass cycles of cultivation-harvest-conversion-degrade-regeneration do not bring alterations to the natural carbon cycle (Johnson, 2009).

An increase in energy crops and forest biomass, i.e. corn, rapeseed, sugarbeet, woodchips, wood pellets, could lead to cropland expansion directly (when new cropland is created), or indirectly (when existing cropland is diverted). Indirect Land-Use Change (ILUC) emissions are assigned to energy crops and forest biomass, according to the factors suggested by The European Parliament and the Council of the European Union (2015) and Ecofys, IIASA and E4tech (2015) for European feedstocks (Figure 5), whose accounting reflects the net impact on the terrestrial carbon pools. It is assumed that energy crops grow in land previously destined to food and feed crops, whose demand is satisfied by bringing non-agricultural land into production elsewhere, thus resulting in indirect land-use change. Land-use changes have impacts on carbon stocks on land, due to loss of above and below ground living biomass and soil organic carbon, but also on biodiversity, land-use rights and other socioeconomic implications (Van Stappen, Brose, and Schenkel, 2011). If land with high carbon stock is converted (e.g. tropical forest and peatland drainage outside the European Union for oil crops), it can lead to significant greenhouse gas (GHG) emissions. For woody biomass, it is assumed it comes from thinning and forest intensification, and there is no land-use change per se; however, there are emissions resulting from a lower build-up of soil organic carbon (Ecofys, IIASA and E4tech, 2015).

IPCC guidelines recommend using country-specific validated data, if possible (IPCC, 2006). However, as this was outside the scope of our analysis, average ILUC values used herein should be regarded as a likely source of uncertainty. Furthermore, ILUC factors have been assumed constant for the whole temporal scope of the analysis, although they will depend on the global demand of biomass for energy and land governance, among other factors, thus possibly varying across time. In this study, no ILUC emissions have been associated to biofuels, which are imported from abroad.
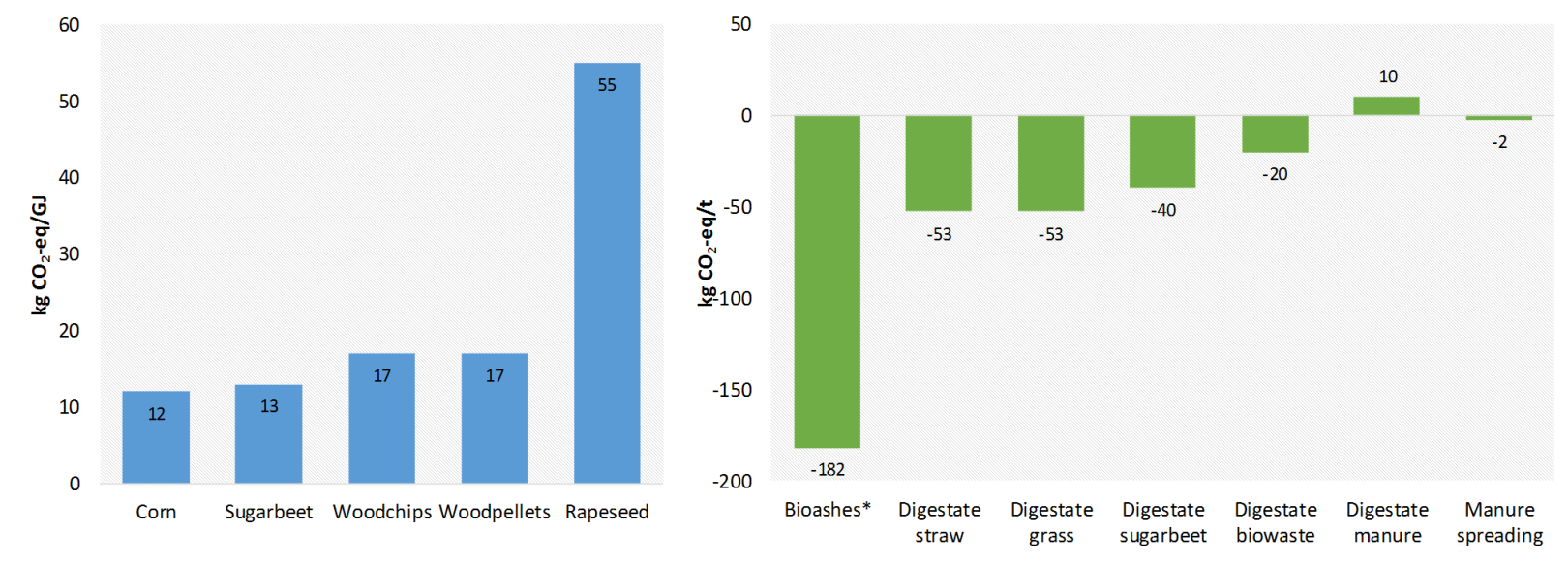

Figure 5: ILUC effects of first-generation biomass [kg $\mathrm{CO}_{2}$-eq/GJ] (left) and carbon emissions from the recycling of biomass into soil $\left[\mathrm{kg} \mathrm{CO} \mathrm{CO}_{2}\right.$-eq/t] (right) annualised over 20 years. Corn, sugarbeet, rapeseed from The European Parliament and the Council of the European Union (2015); woodchips and wood pellets from Ecofys, IIASA and E4tech (2015); bioashes from Sigurjonsson, Elmegaard, Clausen, and Ahrenfeldt (2015); raw manure and digestate (co-digested with manure) from Hamelin, Naroznova, and Wenzel (2014).

*Expressed in $\mathrm{kg} \mathrm{CO}_{2}$-eq/t of straw gasified.

For residual biomass, i.e. animal manure, grass, straw and organic waste, we assume no generation of ILUC emissions, as their utilisation does not lead to additional demand for land. Fugitive losses from biogas production, resulting in $\mathrm{CH}_{4}$ emissions to the atmosphere, have been assumed to be $1 \%$ of the generated methane in volume terms (Hamelin, Naroznova, and Wenzel, 2014), due to the large global warming potential of methane.

Some biomass conversion processes can induce changes in the soil carbon balance, through application 
of digestate from biogas plants, spreading of raw manure on fields and return of bioashes from biomass gasification to land. These considerations are included in the modelling framework, through the assignment of negative carbon emission factors (Figure 5), except for spreading of digestate from manure mono-digestion, which causes net positive $\mathrm{CO}_{2}$ emissions. When ploughing straw in the field, $99.9 \%$ of the carbon is released to the atmosphere over a 20-year period (Hamelin, Naroznova, and Wenzel, 2014), hence straw incorporation contributes only in minor part to negative $\mathrm{CO}_{2}$ emissions, and has thus been considered negligible. For raw manure, it is assumed that it is stored both indoor and outdoor prior to spreading on field. The resulting methane emissions from storage, amounting to $95 \mathrm{~kg} \mathrm{CO}$-eq/GJ of manure (Hamelin, Naroznova, and Wenzel, 2014), thus counterbalance the carbon sequestration effect from soil application depicted in Figure 5.

With respect to fertilising effects, some technologies considered for the utilisation of straw produce soil fertilisers: nitrogen $(\mathrm{N})$, phosphorus $\left(\mathrm{P}_{2} \mathrm{O}_{5}\right)$ and potassium $\left(\mathrm{K}_{2} \mathrm{O}\right)$, with different NPK ratios. Ashes resulting from the gasification of biomass contain soil fertilisers (Sigurjonsson, Elmegaard, Clausen, and Ahrenfeldt, 2015). Similarly, the anaerobic digestion process transforms the biomass into biogas and digestate, the latter having soil fertilising properties (Hamelin, Naroznova, and Wenzel, 2014). The fermentation of ligno-cellulosic material, such as straw, yields bioethanol and lignin, which cannot be converted into sugars with the current technology. Although lignin may have fertilising properties, we herein assumed that it is used for power and heat production (Evald, $\mathrm{Hu}$, and Hansen, 2013). To compare all the different uses of straw, the avoided fertilisers production resulting from ploughing the straw and not removing it from the fields is also accounted for, as well as the possibility of using raw manure directly as fertiliser without undergoing anaerobic digestion (Hamelin, Naroznova, and Wenzel, 2014).

The marginal fertilisers considered for nitrogen, phosphorus and potassium are respectively urea, diammonium phosphate (DAP) and potassium chloride. As decisions on investment and operation of plants follow a least-cost optimization in TIMES-DK, a reward ( $€ / \mathrm{t}$ of fertiliser) is assigned to the avoided fertiliser production for each process (World Bank Group, 2016).

\section{Data}

The study performs a long-term socio-economic optimization of the Danish energy system, with exogenous assumptions on price developments, technology potentials and decommissioning (i.e. shortterm scrapping plans for obsolete facilities). Exogenous future fuel prices are aligned with Danish projections (Danish Energy Agency, 2017b), while electricity prices regulating the trade with neighbouring countries (only used in TIMES-DK), as well as transmission lines capacities, are based on data from the Danish Transmission System Operator (TSO) (Energinet, 2018) and the European TSO (ENTSO-E, 2018). The socio-economic analysis herein performed includes the total costs of satisfying the end-use energy demands under carbon and resource constraints, thereby excluding taxes, levies, subsidies or any other market mechanism for regulation or allocation, and without directly accounting for externalities, except for GHG emissions and soil fertilising effects. Across the analysed scenarios, the same projections apply with regard to end-use demands, technologies characteristics and exogenous prices. Particularly for the latter, it is assumed that the impact of Denmark on fuel prices would be marginal; therefore, these do not change across the analysed scenarios.

This section provides an overview of the technology characterisation for this study, including alternative pathways for the utilisation of straw (Section 3.1) and assumptions on the modelling of biomass resources (Section 3.2). All monetary values are expressed in $€ 2016$.

\subsection{Technology data}

Our model framework includes several technologies for the production of heat, power and fuels. The supply sector comprises the activities related to import, export and extraction of primary energy resources, both fossil and non-fossil. The procured primary energy commodities are transformed into secondary energy commodities, i.e. oil products, biofuels, biogas and hydrogen, through conventional 
crude oil refineries, biorefineries, anaerobic digestion plants and hydrogen production technologies. Some of these technologies produce electricity and/or heat, which can be used for DH, as a by-product in addition to their primary output. Carbon Capture and Storage (CCS) options are currently not considered within the model technology database. For a complete overview of the technology characterisation of biorefineries and biogas plants, the reader can refer to the Supplementary Material A, while Balyk, Andersen, Dockweiler, Gargiulo, Karlsson, Næraa, Petrović, Tattini, Termansen, and Venturini (2019) describes the main data sources used in TIMES-DK.

In the adopted model framework, straw can be utilised in different processes within the energy and agriculture system (Figure 6): a) ploughed in the field, b) production of biogas through anaerobic co-digestion with manure, c) production of bioethanol through hydrolysis and fermentation, d) production of heat in heat-only boilers, e) production of heat and power in combined heat and power (CHP) plants, and f) production of bioalcohols and biodiesel through gasification and Fischer-Tropsch (FT) synthesis, with or without hydrogen addition.

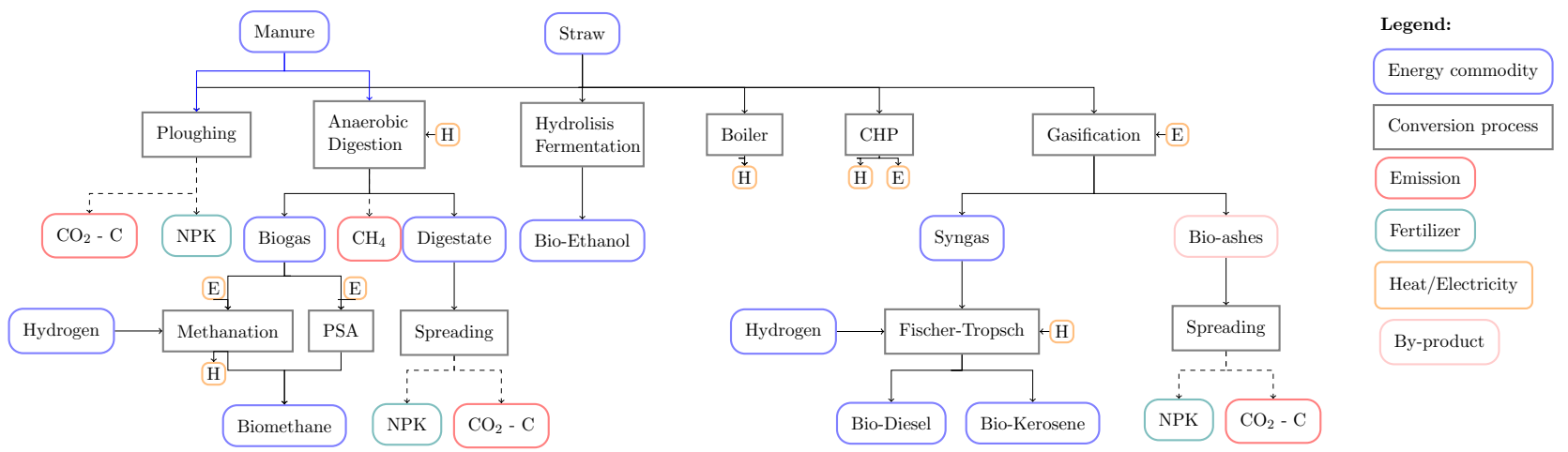

Figure 6: Alternative pathways for the use of straw. CHP $=$ Combined Heat and Power, $\mathrm{H}=\mathrm{Heat}$, $\mathrm{E}=\mathrm{Electricity,}$ PSA $=$ Pressure Swing Adsorption, NPK = Marginal fertilisers (Nitrogen, Phosphorus and Potassium).

As biorefineries produce a considerable amount of excess heat as by-product, we assume that all plants will be built only in central DH areas, thus having a large plant scale, in the range of 800-1400 kt of biomass feedstock per year. On the other hand, biogas plants are located in decentral areas to benefit from the greater availability of the primary input resources, i.e. manure and other agriculture residues. As these resources have high water content, their transportation must be minimised. Moreover, we distinguish between three different sizes of biogas plants to take economies of scale into account (Nielsen and K. Jacobsen, 2017), (Boldrin, Baral, Fitamo, Vazifehkhoran, Jensen, Kjærgaard, Lyng, van Nguyen, Nielsen, and Triolo, 2016): small, medium and large scales respectively correspond to 110, 320 and $500 \mathrm{kt}$ of biomass feedstock per year. The plant investment cost and the transport cost of biomass from the field to the plant are differentiated by plant scale.

\begin{tabular}{lcccc}
\hline Biomass type & Price $(€ /$ GJ) & \multicolumn{3}{c}{ Transport cost } \\
\cline { 3 - 5 } & & Small & Medium & Large \\
\hline Corn & 9.3 & 27.2 & 27.2 & 27.2 \\
Grass & 5.5 & 3.8 & 6.2 & 11.1 \\
Manure & 0.0 & 5.1 & 6.6 & 7.4 \\
Organic waste & 0.0 & 12.5 & 20.6 & 36.9 \\
Rapeseed & 17.7 & 27.2 & 27.2 & 27.2 \\
Straw & 5.5 & 3.8 & 6.2 & 11.1 \\
Sugarbeet & 5.7 & 4.1 & 6.3 & 8.0 \\
Woodchips \& wood waste & 6.1 & 32.2 & 32.2 & 32.2 \\
\hline
\end{tabular}

Table 1: Gate prices (2016) and transport costs of biomass per plant size.

The transport cost of biomass is calculated considering the average transport distance for each plant size, the transport cost by diesel truck, and the loading \& unloading costs. Costs are derived from Joint Research Centre (2015). For each feedstock, a distance radius to the plant is assumed: 23, 
44 and $61 \mathrm{~km}$ for sugarbeet (Boldrin, Baral, Fitamo, Vazifehkhoran, Jensen, Kjærgaard, Lyng, van Nguyen, Nielsen, and Triolo, 2016); 6, 8 and $10 \mathrm{~km}$ for manure (Boldrin, Baral, Fitamo, Vazifehkhoran, Jensen, Kjærgaard, Lyng, van Nguyen, Nielsen, and Triolo, 2016); and 5, 10 and $20 \mathrm{~km}$ for the other biomass types. The resulting transport costs for the different biomass feedstocks ( $€ € / G J)$ are reported in Table 1, along with the biomass price seen at the gate $(€ / \mathrm{GJ})$.

Figure 7 compares the total cost ( $€$ /GJ biogas) and energy efficiency of the biogas plants considered in this study. When straw is anaerobically co-digested with manure to produce biogas, a pre-treatment step is added to enhance the hydrolysis phase and ultimately the biogas yield, by increasing the recovery of sugars from the ligno-cellulosic material (Kurian, Nair, Hussain, and Raghavan, 2013). Several pre-treatment options are commercially available, including mechanical, chemical, biological and thermo-chemical treatment. We assumed that straw is pre-treated through mechanical extrusion (Zheng and Rehmann, 2014), based on Danish experiences for biogas production, at a cost of $4.0 € /$ GJ of straw (Danish Energy Agency, 2014). Also sugarbeet needs to undergo pre-treatment, i.e. washing, slicing and ensiling, prior to the anaerobic co-digestion with manure. The pre-treatment cost is assumed at $4.5 € /$ GJ of sugarbeet, as proposed in the literature relevant for the Danish case (Boldrin, Baral, Fitamo, Vazifehkhoran, Jensen, Kjærgaard, Lyng, van Nguyen, Nielsen, and Triolo, 2016).

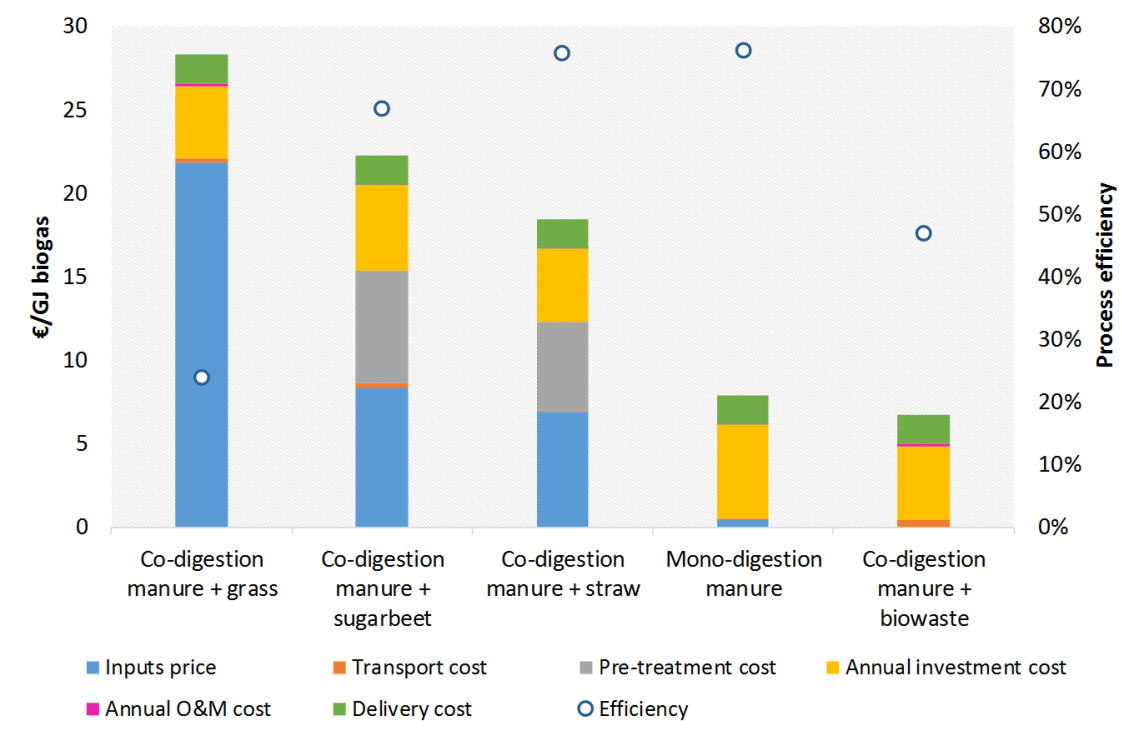

Figure 7: Total cost of production of biogas by plant type (medium scale plants are assumed). Transport costs cover the transport of biomass feedstocks to the plant, while delivery costs refer to the transport of biogas to the final use. Investment costs are annualised with a $4 \%$ discount rate and 40 years lifetime, and reported for the year 2016.

\subsection{Availability of resources}

Spatial availability of energy resources can vary greatly, depending on the location and density of residential and industrial settlements, agriculture fields, forest and farming areas. Moreover, while temporal availability of biomass largely depends on seasonal and annual variations affecting farming and natural forests, the generation of heat from biorefineries and industries is primarily driven by the need to utilise the invested production assets optimally. In addition, waste incineration currently allows for the safe disposal of residual waste in Denmark, where landfilling of combustible waste is banned. As waste storage is constrained, considering e.g. the large share of organic waste in the residential fraction, coupled with the need to limit plant over-dimensioning due to high capital costs, waste incineration provides a rather constant delivery of power and heat. Accounting for variation in the temporal and geographical availability of resources can provide a more detailed picture of their competing uses, as well as the value of the by-products provided. This subsection describes the assumptions behind the representation of biomass (Section 3.2.1), residual waste (Section 3.2.2) and industrial excess heat (Section 3.2.3) in our modelling framework. 


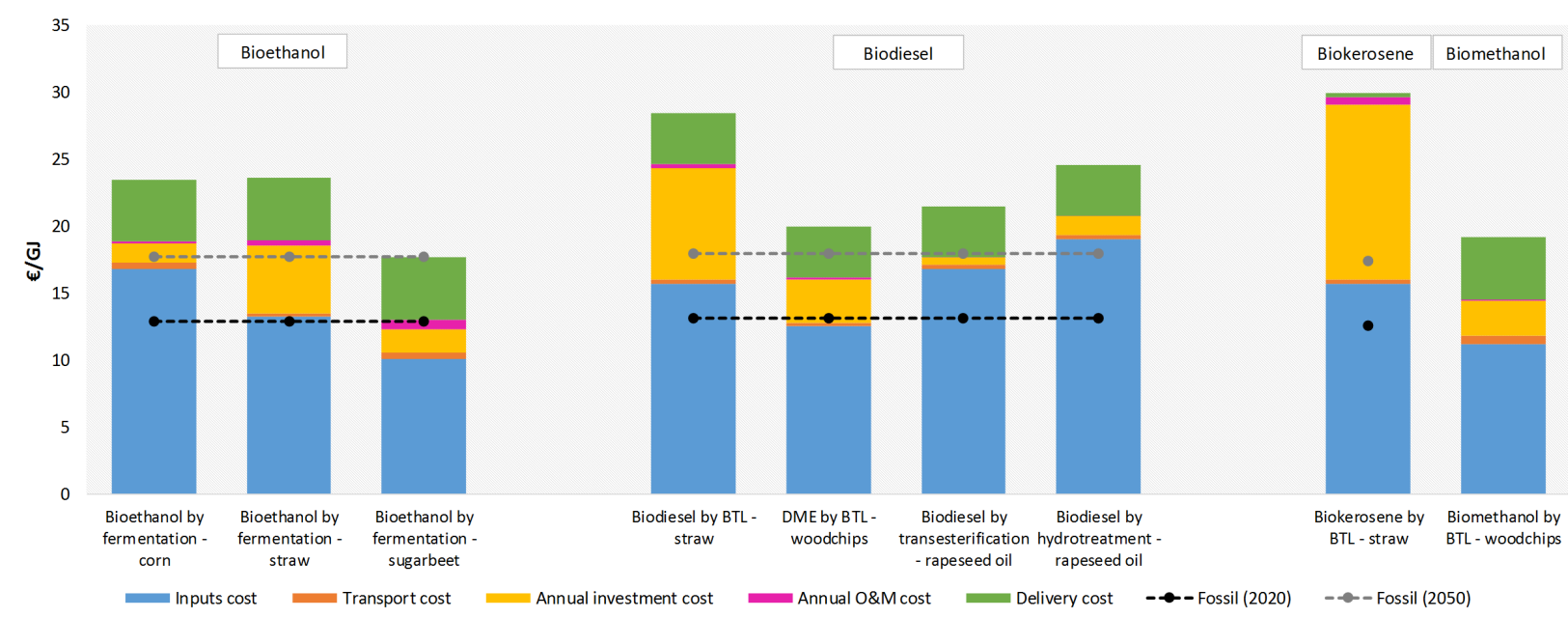

Figure 8: Total production cost (€/GJ fuel) for range of biofuels which can be domestically produced, compared to fossil counterparts (respectively gasoline, diesel and biokerosene) for 2020 and 2050. Transport costs cover the transport of biomass feedstocks to the plant, while delivery costs refer to the transport of biogas to the final use. Investment costs are annualised with a $4 \%$ discount rate and 20 years lifetime.

\subsubsection{Biomass resources}

The biomass resources considered in this study are manure, grass, straw, organic waste, corn, rapeseed, sugarbeet, wood pellets, woodchips and wood waste, as described in Table 2. Some of these feedstocks could be imported from abroad, when the demand exceeds the national supply. Potentials have been estimated considering the amount of biomass which is technically available under business-as-usual conditions, e.g. assuming no changes in diet, crop production and land management practices (Tonini, Vadenbo, and Astrup, 2017). The manure potential is expected to rise by 2050 without a corresponding increase in its generation rate, due to increased collection for anaerobic digestion. Third-generation biomass, e.g. algae, is not included in this study.

\begin{tabular}{|c|c|c|c|c|}
\hline \multirow[t]{2}{*}{ Feedstock } & \multicolumn{2}{|c|}{ Potential (PJ) } & \multirow[t]{2}{*}{ Domestic/Imported } & \multirow[t]{2}{*}{ Reference } \\
\hline & 2015 & 2050 & & \\
\hline Corn & 45 & 45 & D \& I & Danish Energy Agency (2013) \\
\hline Grass & 4.1 & 6.7 & $\mathrm{D}$ & Danish Energy Agency (2014) \\
\hline Manure & 14.5 & 28.7 & $\mathrm{D}$ & Danish Energy Agency (2013), AgroTech (2013) \\
\hline Organic waste & 1.9 & 4.2 & $\mathrm{D}$ & Andersen and Larsen (2012) \\
\hline Rapeseed & 10 & 15 & D \& I & Danish Energy Agency (2013) \\
\hline Sugarbeet & 10 & 15 & D \& I & Danish Energy Agency (2013) \\
\hline Straw & 54 & 54 & $\mathrm{D}$ & Statistics Denmark (2016) \\
\hline Woodchips \& wood waste & 40.7 & 41.6 & $\mathrm{D} \& \mathrm{I}$ & Danish Energy Agency (2013) \\
\hline Wood pellets & $\mathrm{N} / \mathrm{A}$ & $\mathrm{N} / \mathrm{A}$ & I & - \\
\hline Total domestic & 176.5 & 206.1 & & \\
\hline
\end{tabular}

Table 2: Domestic (D) biomass potentials (PJ). D indicates that the resource is available in Denmark, while I indicates that the resource can be imported from abroad.

As this study aims at evaluating the optimal use of straw, including the location and capacity of biorefineries, we conducted a detailed geographical assessment of its availability. The generation of straw is mapped considering the agricultural areas of the crops that produce the largest amounts of straw in Denmark: winter wheat, spring barley, rye, winter barley and oats, which account for $97 \%$ of the total straw generation (Danish Agricultural Agency, 2016). The amount of straw generated at a national level is calculated assuming the national average straw yield per hectare of specific cropland, as reported in Statistics Denmark (2016) (see Supplementary Material B for further information). The straw available is calculated as the percentage of straw currently used for energy, plus the fraction left-on-field (Figure 9), as proposed by Tonini, Hamelin, and Astrup (2016). Therefore, the amount of straw currently destined to animal bedding and fodder is excluded from the potential. 


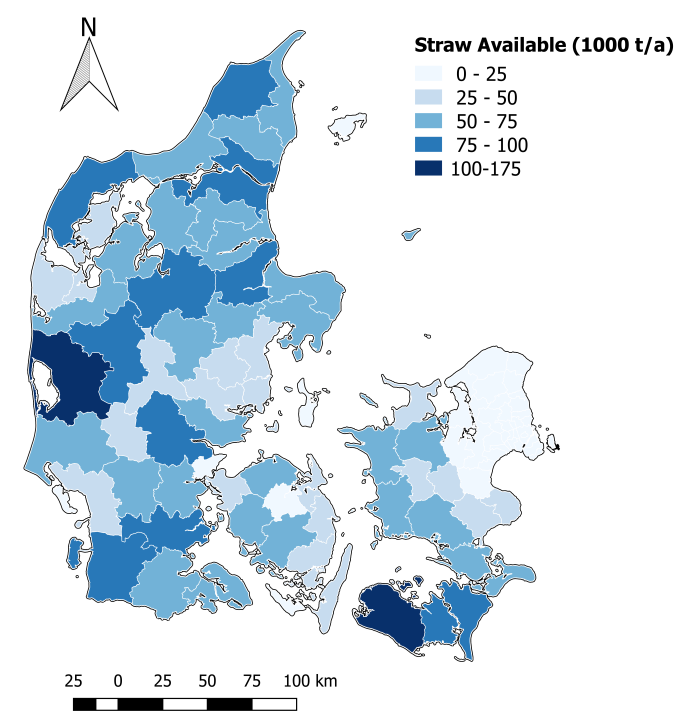

Figure 9: Straw available for energy in Denmark in 2016, aggregated at municipality level.

However, the resulting potential should be regarded with caution, as there is yet a need to assess the amount of straw that could be safely removed without incurring in soil deterioration and carbon loss (Blanco-Canqui and Lal, 2009). For instance, Hamelin, Jorgensen, Petersen, Olesen, and Wenzel (2012) showed that straw removal can affect the carbon balance, depending on the crop and the type of soil. On the other hand, allocating straw to energy purposes might be preferred over straw ploughing when pursuing climate mitigation goals (Powlson, Riche, Coleman, Glendining, and Whitmore, 2008), (Monteleone, Cammerino, Garofalo, and Delivand, 2015).

Straw is generated and stored after harvesting, which is a seasonal process. Changes in the composition of straw during storage are relatively small. Hamelin, Naroznova, and Wenzel (2014) report a loss of $1.1 \%$ of the initial dry matter content after storage of dry straw bales for eight months. Therefore, it is assumed that storage does not alter the composition, and annual potentials (PJ/year) are thereby considered, i.e. straw must be used within a year of its generation. Finally, it is assumed that the potential of straw available for energy in Denmark does not vary across time, i.e. it is equal to the amount estimated for 2016.

\subsubsection{Residual waste}

Residual waste comprises combustible waste from households, services and industry, which does not undergo a recycling process and needs to be disposed. In Denmark, $54 \%$ of the residual waste was incinerated in 2014 for energy recovery (Eurostat, 2018). Although new options for residual waste treatment might be developed in the coming years, with a higher added-value in economic and/or environmental terms, e.g. gasification (Pizarro-Alonso, Cimpan, and Münster, 2018b), this study assumes that all the residual waste available in Denmark has to be incinerated. Residual waste must be incinerated within a week of its collection, while up to $50 \%$ of industrial waste can be stored for a year.

Waste amounts, along with their composition, vary over time as a result of changes in, e.g. population, consumption patterns, GDP and recycling rates. The econometric tool FRIDA is used to estimate the amount and fractions of waste generated at a national level until 2050 (Andersen and Larsen, 2012). The amount of residual waste is calculated by defining specific recycling goals for each fraction, as to comply with the EU target setting the recycling of waste to minimum $65 \%$ by 2030 (European Commission, 2017), herein maintained until 2050, as shown in Figure 10.

The availability of organic waste for anaerobic co-digestion with manure is expected to increase from 1.9 to $4.2 \mathrm{PJ}$ (Table 2). This is due to an increase in waste separation at the source of generation, which is a requirement to ensure the traceability of the waste, as well as the further possibility to spread digestate on-land as a fertiliser (Danish Ministry of the Environment and Food, 2018). 


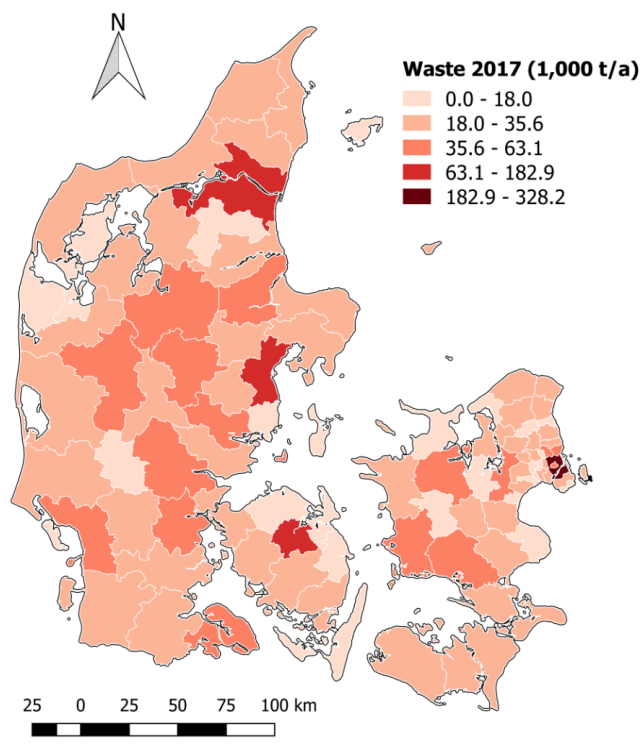

(a) Current geographical distribution

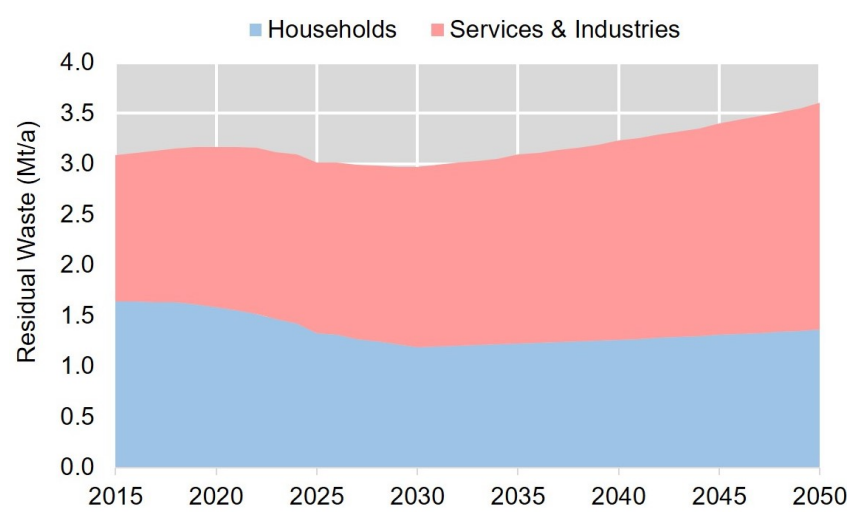

(b) Future prognosis

Figure 10: Geographical distribution of residual waste (a) and residual waste prognosis for Denmark (b).

\subsubsection{Industrial excess heat}

Bühler et al. $(2017,2016)$ have highlighted the unexploited potential for excess heat recovery from thermal processes in Denmark after evaluating 22 industrial sectors, representing $79 \%$ of the energy used in the Danish industry. Our study considers the excess heat production available within the large district heating areas, i.e. $2.2 \mathrm{PJ} / \mathrm{a}$, and the possibility to invest in heat pumps to utilise the potential from low-heat sources, which could provide up to $2.1 \mathrm{PJ} / \mathrm{a}$, as illustrated in Figure 11. Furthermore, we assume that the amount of industrial excess heat and its geographical availability will not vary throughout the years, as to reflect that no major changes will occur in the industry composition and facilities location.

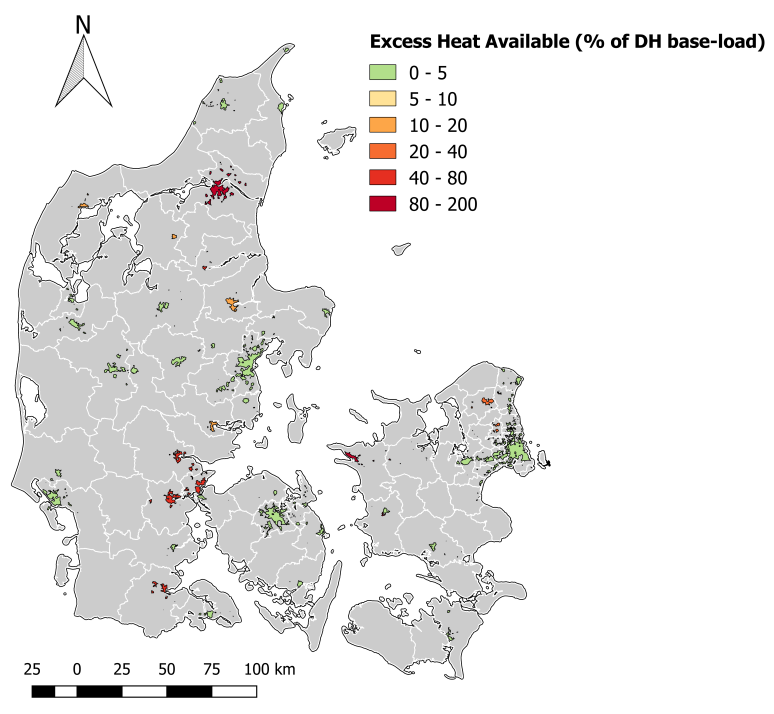

Figure 11: Geographical distribution of exploitable industrial excess heat in Denmark in 2014 (adapted from Bühler, Petrovic, Karlsson, and Elmegaard (2017) and Bühler, Nguyen, and Elmegaard (2016)). 


\section{Scenario definition}

To investigate the optimal allocation of straw to the alternative uses under different conditions, three scenarios are herein analysed, as described in Table 3. The scenarios progressively examine the cumulative impact of different constraints on the use of biomass, while modelling the whole energy system. All scenarios include a constraint on the share of first-generation biofuels: in accordance with the Renewable Energy Directive of the European Commission (European Parliament, 2018), (European Commission. Directorate-General for Climate Action, 2016), the contribution from biofuels produced from food or feed crops will be no more than $7 \%$ of the final energy consumption in road and rail transport by 2020. This limit is further reduced to $3.8 \%$ by 2030 (European Commission. Directorate-General for Climate Action, 2016).

\begin{tabular}{|c|c|c|c|c|c|c|c|}
\hline Scenario & Abbreviation & Straw use & Manure use & $\mathrm{CO}_{2}$ Target & Biomass imports & Biofuels imports & Biofuel blending \\
\hline Business As Usual & BAU & $\begin{array}{c}\text { Heat and Power } 23 \% \\
\text { Left on field } 50 \% \\
\text { Fodder and bedding } 27 \%\end{array}$ & - & & Allowed & Allowed & $\mathrm{X}$ \\
\hline Carbon-Constrained & $\mathrm{CO} 2$ & $\begin{array}{c}\text { Energy system } 73 \% \\
\text { Fodder and bedding } 27 \%\end{array}$ & - & $\mathrm{X}$ & Allowed & Allowed & $\mathrm{X}$ \\
\hline No bioenergy imports & NO-IMP & $\begin{array}{c}\text { Energy system } 73 \% \\
\text { Fodder and bedding } 27 \%\end{array}$ & $\geq 50 \%$ & $\mathrm{X}$ & Not allowed & Not allowed & $\mathrm{X}$ \\
\hline
\end{tabular}

Table 3: Scenarios definition. $\mathrm{X}$ indicates active constraint.

The Reference scenario replicates the current use of straw (Statistics Denmark, 2016) until the end of the modelling horizon. The split on the use of straw is calculated on the basis of its energy content (\% PJ) in 2014: $23 \%$ of the total harvested straw was utilised in the heat and power sector, i.e. CHP plants and heat-only boilers, $27 \%$ was destined to fodder and bedding for animals, while the remaining $50 \%$ was left on the field. Moreover, in the Reference case, there are no imposed restrictions on imports of biomass from abroad, as to reflect the current policies.

In the Carbon-constrained scenario (CO2), we analyse the effect of imposing a bound on the $\mathrm{CO}_{2}$-eq emissions for the whole energy system, yet excluding waste incineration, as this activity is highly regulated and assumed to continue in the future. Therefore, according to the IPCC (2006) guidelines for National Greenhouse Gas Inventories, Eq.1 includes all the domestic emissions associated to stationary and mobile combustion (category 1A); and some emissions from agriculture, forestry and land use (categories 3A2, 3B \& 3D), but only of those resources that could potentially be used for energy purposes in Denmark (see Table 2). The overarching target on $\mathrm{CO}_{2}$-eq emissions for the year 2050 follows from Denmark's ambition of being climate-neutral by 2050, i.e. Denmark absorbs at least as much GHG as it emits (Danish Ministry of Energy, Utilities and Climate, 2018). As a result, we set the sum of $\mathrm{CO}_{2}$-eq emissions $\left(C A P_{y}\right)$ from all sectors $(S)$ to zero for year $y=2050$, including fossil $G H G_{s, y}^{f o s s i l}$, indirect land-use change $G H G_{s, y}^{I L U C}$ and biogenic $G H G_{s, y}^{b i o}$ components, as in Eq.1. Nevertheless, this assumption should be regarded as conservative, as emissions from the agriculture sector in 2015 were $22 \%$ of the total Danish GHG emissions (excluding land use), while the land use, land-use change and forestry (LULUCF) sector was estimated to be a net sink of $1 \%$ of the total GHG emissions (Nielsen, . Plejdrup, Winther, Nielsen, Gyldenkærne, Mikkelsen, Albrektsen, Thomsen, Hjelgaard, Fauser, . Bruun, Johannsen, Nord-Larsen, Vesterdal, Callesen, Caspersen, Rasmussen, Petersen, Baunbæk, and Hansen, 2017). As these two sectors are not accounted in Eq.1, a negative cap might actually be required by 2050 to achieve the desired goal of climate neutrality at a national level, unless emissions from the agricultural sector decrease drastically or the forest carbon sink increases.

$$
\sum_{s \in \text { Sector }} G H G_{s, y}^{\text {fossil }}+G H G_{s, y}^{I L U C}+G H G_{s, y}^{b i o}=C A P_{y}
$$

Additionally, in the CO2 scenario the constraint on the use of straw is relaxed, leaving the costminimisation program free to optimise the use of straw in the energy system considering the different alternative uses: left on field; anaerobically co-digested with manure for the production of biogas; combusted for the production of heat or combined heat and power; treated (hydrolysis) and fermented for the production of bioethanol; gasified at high temperature and subsequently liquefied through 
FT synthesis to produce biokerosene or biodiesel.

The No bioenergy imports scenario $(N O-I M P)$ is centred around the utilisation of domestic resources. Imports of first-generation biomass, i.e. corn, rapeseed, sugarbeet, woodchips and wood pellets, as well as imports of liquid biofuels from outside Denmark follow a progressive decrease along the time horizon until 2050, when imports are no longer allowed. Additionally, to strengthen the role of the agricultural sector as a supplier of green energy (Green Growth Agreement, signed by the Danish Government in 2009 and setting goals for 2020 (The Danish Government, 2009)), we force the use of at least $50 \%$ of the livestock manure potential for the production of biogas.

For all scenarios, the time horizon ranges from 2010 to 2050, split in five-year periods, with 2015 as calibration year.

\section{Results}

The size of a model influences its solving time: the NO-IMP scenario of this study is characterised in TIMES-DK by 801,418 rows, i.e. equations, and 928,343 columns, i.e. variables or endogenous parameters, while the Balmorel \& OptiFlow model is characterised by 2,367,358 equations and 4,400,779 variables. As described in Section 2, TIMES-DK is used as a linear programming model, while Balmorel \& OptiFlow, as applied in this study, is formulated as a mixed integer linear programming problem to model endogenously economies of scales. Therefore, due to the differences in sizes and mathematical formulations, solving times differ significantly, with an average of 8 minutes for TIMES-DK and 720 minutes for Balmorel \& OptiFlow. In both cases, a CPLEX solver with barrier algorithm is used.

\subsection{Transition of the energy system}

The optimal allocation of residual biomass should be analysed in the perspective of the transition from the current to the future Danish energy system (Figure 12). The energy system becomes increasingly integrated, with a growing electrification of the transport sector and heat production in the three scenarios. Electricity and district heating generation are almost fossil-free, except for the fossil component of residual waste, i.e. plastics. Nevertheless, the consumption of fossil fuels for heavy transport and process heating is still significant in the Reference scenario, i.e. 90 PJ in 2050, whereas the oil consumption decreases by $70 \%$ compared to 2015 levels. The CO2 and NO-IMP scenarios (Figure 13 minimise the fossil fuel consumption by an increase in the energy efficiency, the use of biofuels, investments in solar heating for low-temperature process heating in the industrial sector, and the electrification of part of the demand. In addition, due to the limited biomass resources in the NO-IMP scenario, constrained by the domestic potential, hydrogen is used to upgrade biogas and syngas, boosting the production of biofuels. Hydrogen is mostly produced via electrolysis, thereby increasing even more the electricity demand in the NO-IMP scenario, as well as the integration between electricity, heat and transport sectors.

Renewable sources like wind and solar cover the entire domestic power production in 2050, except for waste incineration plants. The increase in electricity demand in the CO2 and NO-IMP scenarios is mostly fulfilled through the growth in offshore wind installations and in transmission capacity with neighbouring regions. Heat generation in central areas shifts away from coal cogeneration plants towards a combination of waste incineration, solar heating and excess heat from biorefineries in the three scenarios. In decentral areas, heat pumps (HP) become the major source of heat supply, providing around $62 \mathrm{PJ}$ of heat in 2050 in the Reference, compared to $20 \mathrm{PJ}$ in 2015. 


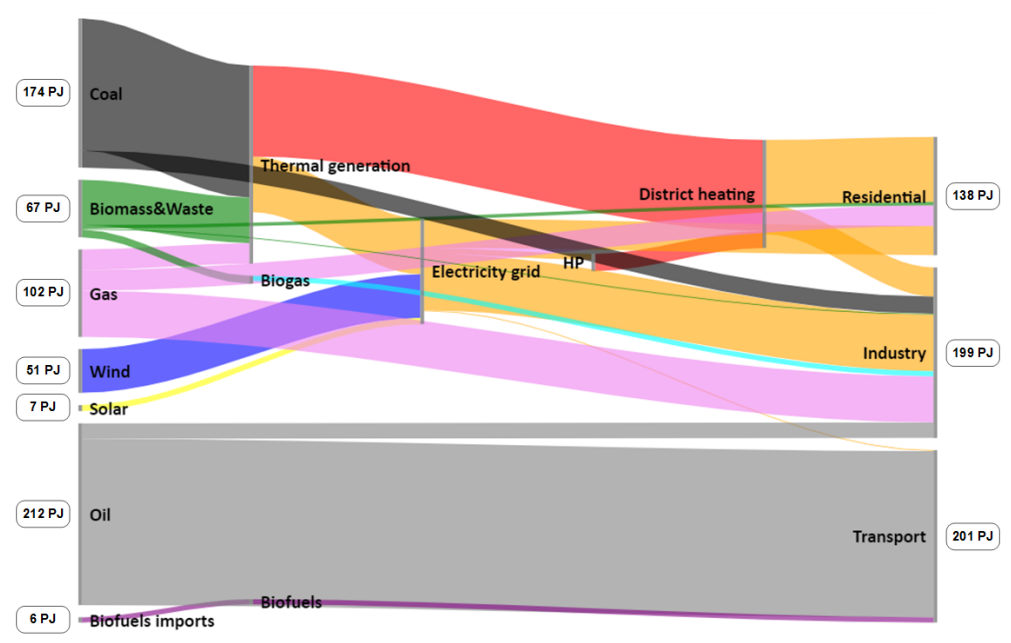

(a) 2015

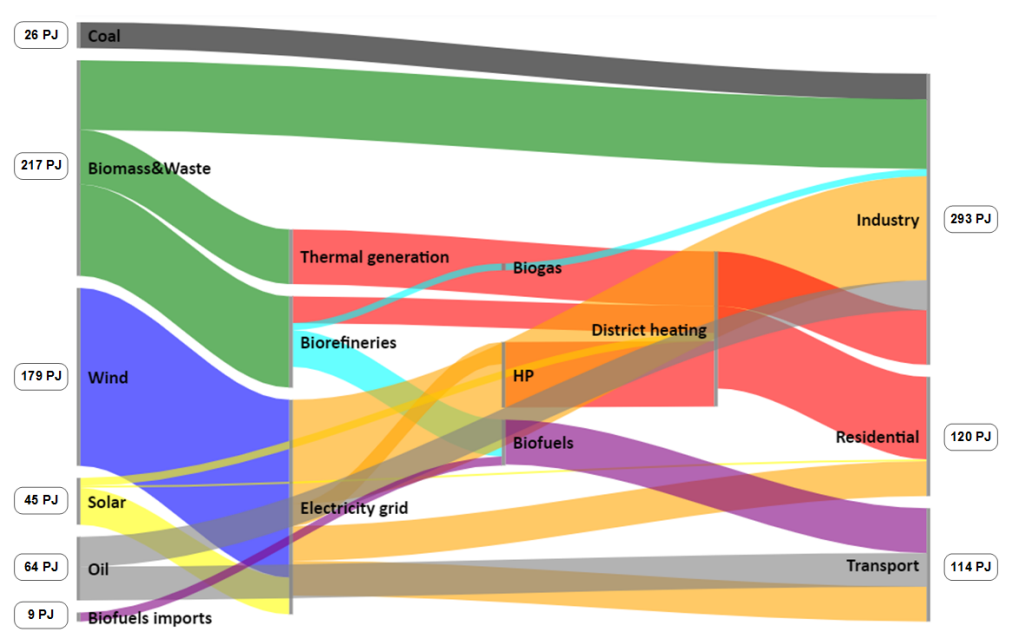

(b) 2050

Figure 12: Danish energy system in the Reference scenario. The Sankey diagram reports the energy flows for the whole energy system, from primary sources (both domestic and imported) to conversion processes (e.g. power plants) to the end-uses (i.e. residential, industry and transport). Electricity trade with neighbouring countries is not reported for simplicity.

\subsection{Allocation of biomass resources}

Biomass and waste play a relatively small role in 2015, accounting for $10 \%$ of the primary energy supply, with 53 PJ used for the generation of heat and power, 9 PJ for the production of biogas, and $5 \mathrm{PJ}$ in the industry and residential sectors. On the other hand, biomass and waste play a much larger role in the energy system in 2050 already in the Reference scenario, corresponding to $40 \%$ of the primary energy supply and replacing a great share of oil, coal and gas: 55 PJ are destined to heat and power production, $70 \mathrm{PJ}$ to industry and $92 \mathrm{PJ}$ are converted to biofuels and biogas for the transport and industry sectors. Biomass and waste play a dominant role also in the CO2 scenario, amounting to $37 \%$ of the total primary energy supply in 2050 , while it has a more modest role in the NO-IMP scenario (23\% of primary energy supply in 2050), due to the constraint on the sole use of domestic resources. Biofuel production increases from 9 PJ in 2015 to 37 PJ in 2050 in the Reference scenario (Figure 14), primarily consisting of biodiesel from gasification of woody biomass and biogas from co-digestion of manure and organic waste, respectively used in freight trucks and industry.

Although the transport sector witnesses an increased level of electrification already in the Reference scenario, corresponding to $31 \%$ of the total fuel consumed by all modes in 2050, sustainable liquid or gaseous fuels are needed for those modes, such as heavy transport, navigation and aviation, where electrification might be more difficult to achieve (with $36 \%, 32 \%$ and $1 \%$ supplied by respectively 


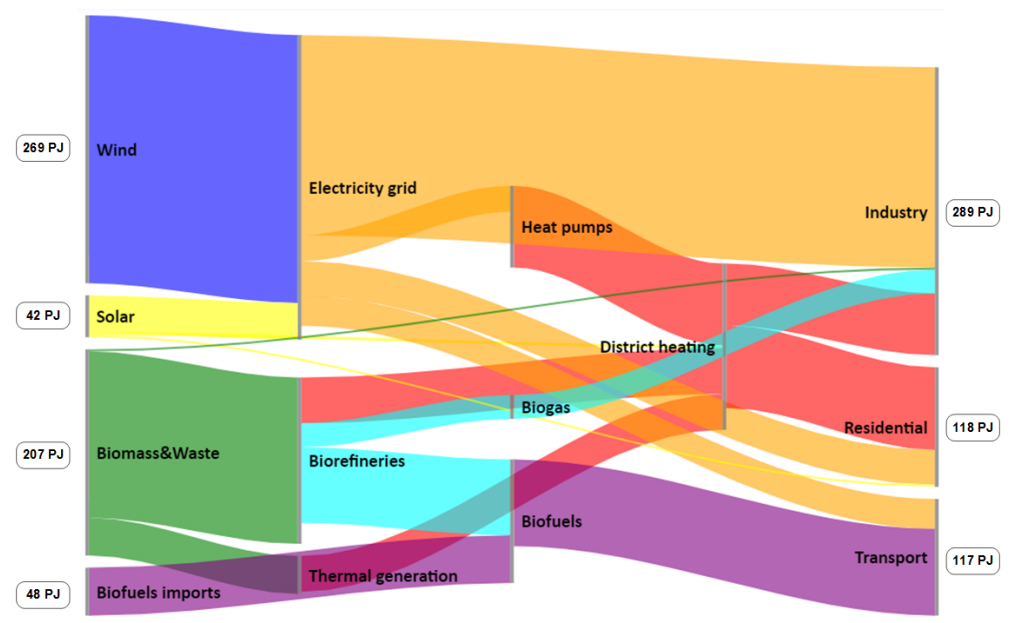

(a) $\mathrm{CO} 2(2050)$

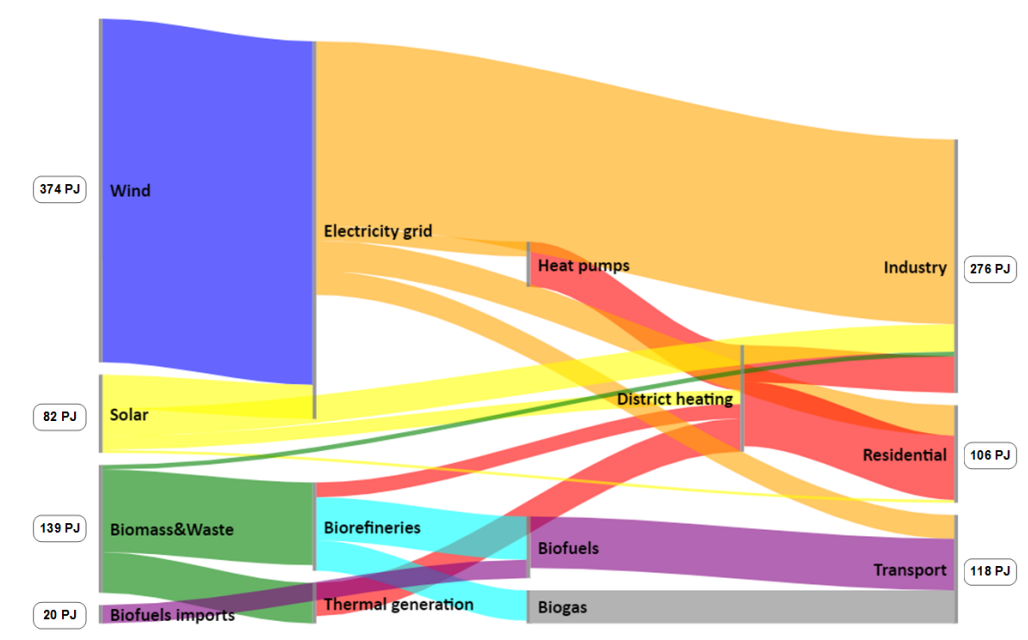

(b) NO-IMP (2050)

Figure 13: Danish energy system in the CO2 and NO-IMP scenarios, for the year 2050.

(bio)kerosene, biodiesel and heavy fuel oil in the Reference scenario in 2050). In the CO2 scenario, biodiesel from gasification of straw and woody biomass amounts to 76 PJ in 2050, destined to industry, freight trucks and national shipping. Around 73 PJ of woodchips or wood pellets need to be imported in order to meet the demand for fuel and industrial heat.

In the CO2 and NO-IMP scenarios, biogas production increases considerably by 2050 due to the need of avoiding the application of raw manure on land, as constrained by the climate-neutrality equation, as well as to maximise the value of domestically available resources in the NO-IMP scenario.

The utilisation of straw changes across the three scenarios analysed, as illustrated in Figure 15. In the Reference case, straw use reflects the current shares, with around 36 PJ left for ploughing on agriculture fields, and 18 PJ combusted in CHP plants and heat-only boilers. In the CO2 scenario, the imposition of a bound on $\mathrm{CO}_{2}$ emissions drives the allocation of straw away from agriculture fields towards biorefineries to produce biofuels, which displaces the use of fossil fuels in the energy system. Starting from 2025, straw is increasingly converted to biodiesel through gasification and FT synthesis. In the most stringent scenario, $N O-I M P$, where imports of bioenergy from abroad are not allowed, straw is mostly converted to biofuels for aviation, i.e. biokerosene.

In the NO-IMP scenario, almost all biogas produced in 2050 (33 PJ - see Figure 14) from codigestion of manure, grass, organic waste and energy crops is upgraded to natural gas quality through methanation with hydrogen, which allows to boost the methane production. Biomethane supplies part of the heavy-duty road transport with domestic bioenergy resources, while the remaining 0.3 PJ are used in industrial sectors for high-temperature process heating. Furthermore, both biodiesel 


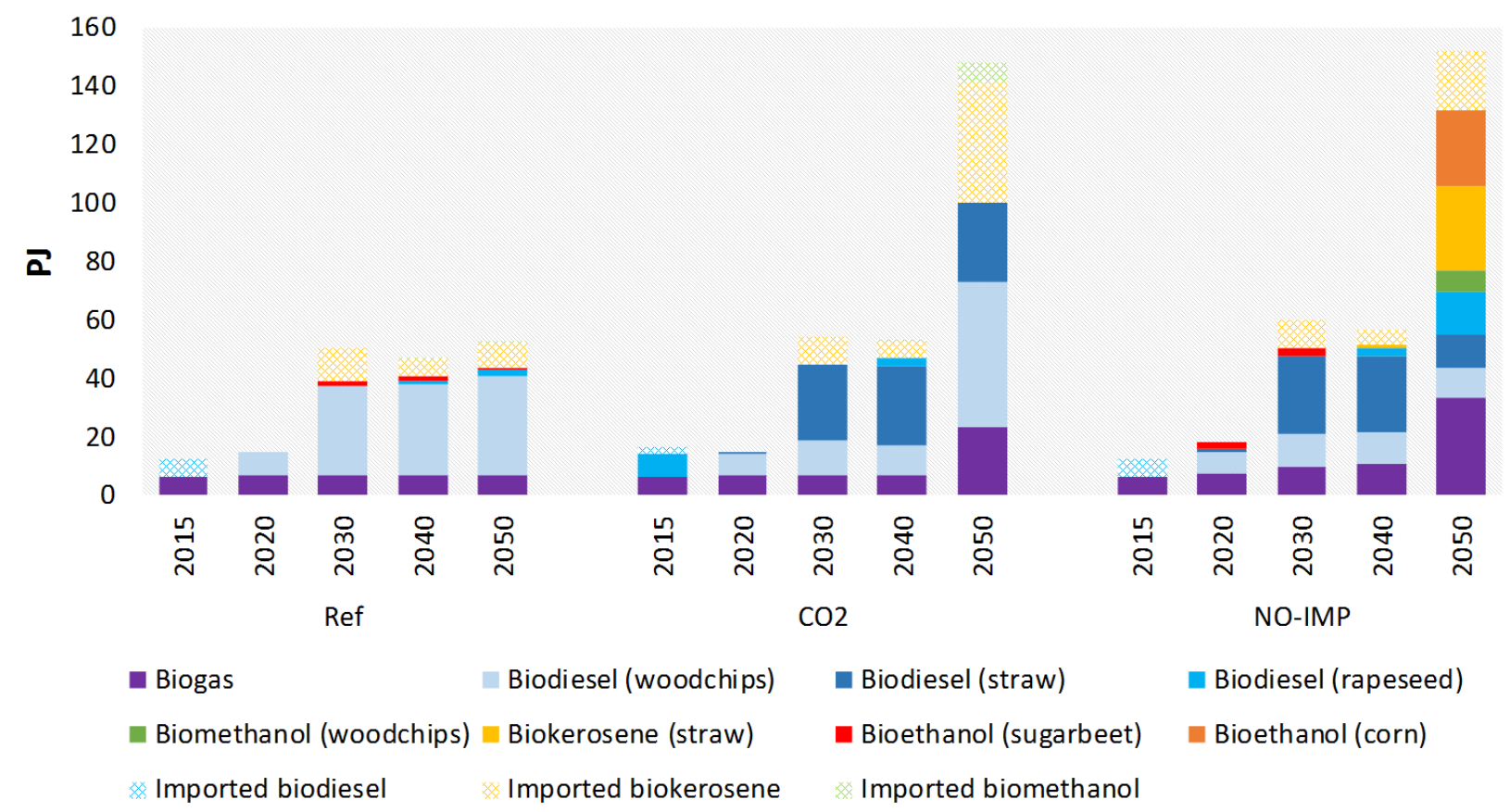

Figure 14: Biofuel and biogas production and imports in the analysed scenarios.

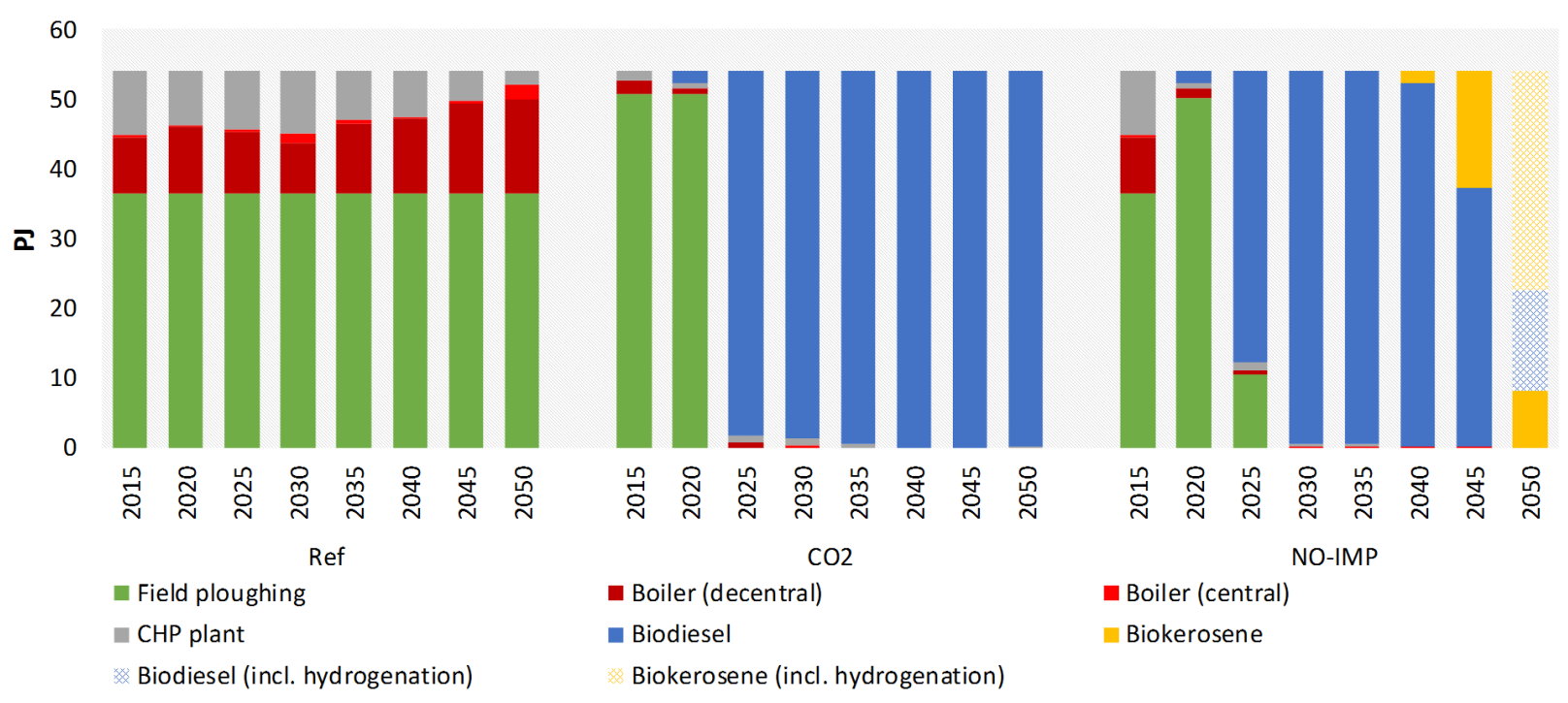

Figure 15: Use of straw across years in the analysed scenarios.

and biokerosene production processes use hydrogen from electrolysis (31 $\left.\mathrm{PJ} \mathrm{H}_{2}\right)$ to boost the process efficiency (Figure 15). Therefore, it is only under the condition of restricting bioenergy imports, thus relying solely on domestic resources, that hydrogen plays a stronger role in the energy system. However, this last scenario presents a certain degree of infeasibility, suggesting that domestic resources are not sufficient to supply the whole final energy demands. Imports of biofuels will be still required in 2050, e.g. 20 PJ of biokerosene for the aviation sector, unless new technologies are developed or the domestic biomass potential increases through changes in, e.g. land use and agricultural practices, while respecting sustainability principles. The infeasibility of the NO-IMP scenario is detected through the definition of slack variables, simulating the additional required import of resources (at a higher price) to satisfy the end-use demands. The introduction of slack variables allows to detect model infeasibilities while at the same time obtaining an optimal solution for the problem.

In the formulation of the optimization model TIMES-DK, the marginal value of straw, which is the shadow price of the equation that restricts its availability, describes the cost change in the objective 
function if the availability of the commodity is increased by one unit, i.e the benefit or expense associated to the consumption of an additional unit of commodity. Therefore, the marginal value of straw, as shown in Figure 16, gives an indication of its market value under perfect competitive market conditions. The marginal price of straw doubles in the CO2 scenario with respect to the Reference, from 11 to $22 € /$ GJ in 2050 , due to the rise in the demand of biomass to comply with the emission reduction targets. For comparison, the current average Danish price for straw at the plant gate amounts to 5.0-5.5€/GJ (Bentsen, Nilsson, and Larsen, 2018). Under the hypothetical condition of supplying only from domestic biomass resources by 2050 (NO-IMP scenario), the marginal value of straw is affected by the existence of an infeasibility related to the inability to satisfy the whole energy demand with domestic resources, as well as the lack of alternative technologies, e.g. ammonia for shipping (van Biert, Godjevac, Visser, and Aravind, 2016) or electrified roads. This results in an extremely high marginal price of straw ( $2845 € /$ GJ in 2050 ), reflecting the value attributed to the goal of self-sufficiency.

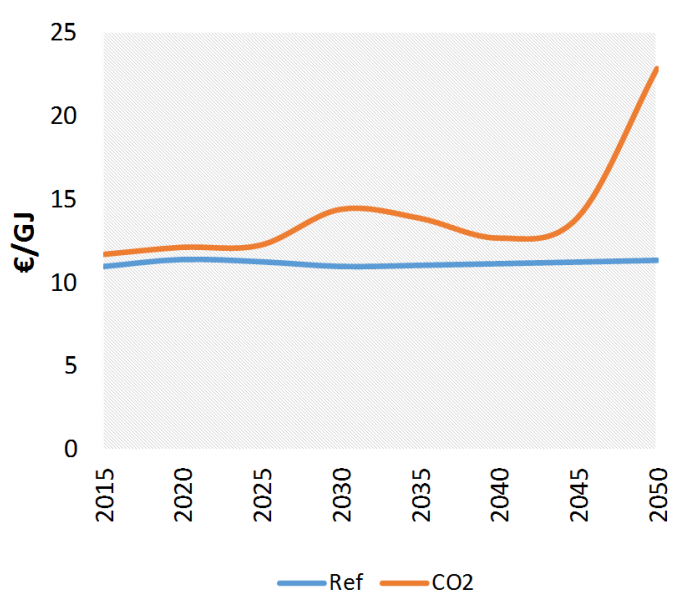

Figure 16: Marginal price of straw in the analysed scenarios.

Figure 17 compares the annual costs of the energy system for the three scenarios, all of which witness increasing investment and fixed O\&M costs over the modelled years. Moreover, to more stringent policies correspond higher total costs, as a result of the increasing number of constraints imposed onto the system, i.e. carbon abatement and resource limitations. As a result, the total discounted system cost for the analysed time horizon (i.e. the objective function of the optimization problem) equals to respectively 291, 293 and 483 billion $€$ for the Reference, CO2 and NO-IMP scenarios. The much higher system cost for the $N O-I M P$ case is also to be ascribed to the presence of slack biofuels imports (amounting to 159 billion $€$ ).

\subsection{The role of biorefineries in future energy systems}

Biorefineries play an important role in ensuring the transition towards a climate-neutral system, where the use of fossil fuels is progressively phased out. Furthermore, biorefineries can provide valuable by-products, such as heating, as long as there is a market that can absorb it. Results from Balmorel-OptiFlow in Figure 18 show the optimal location and sizing of biorefineries, constrained by economies of scale, considering the availability of resources and the competition between waste incineration plants and industrial excess heat for supplying the base-load heat.

In 2050, biorefineries may supply up to one third of the DH demand in central areas in Denmark (in the CO2 scenario, where heating production from biorefineries is the largest), if their location as well as investments in inter-seasonal heat storage (e.g. water pitches) are optimized. The installed capacity in inter-seasonal heat storage in central DH networks by 2050 corresponds to $991 \mathrm{GWh}$ (3\% of the total DH demand in those networks) in the Reference, $1356 \mathrm{GWh}$ (4\% of the total DH 


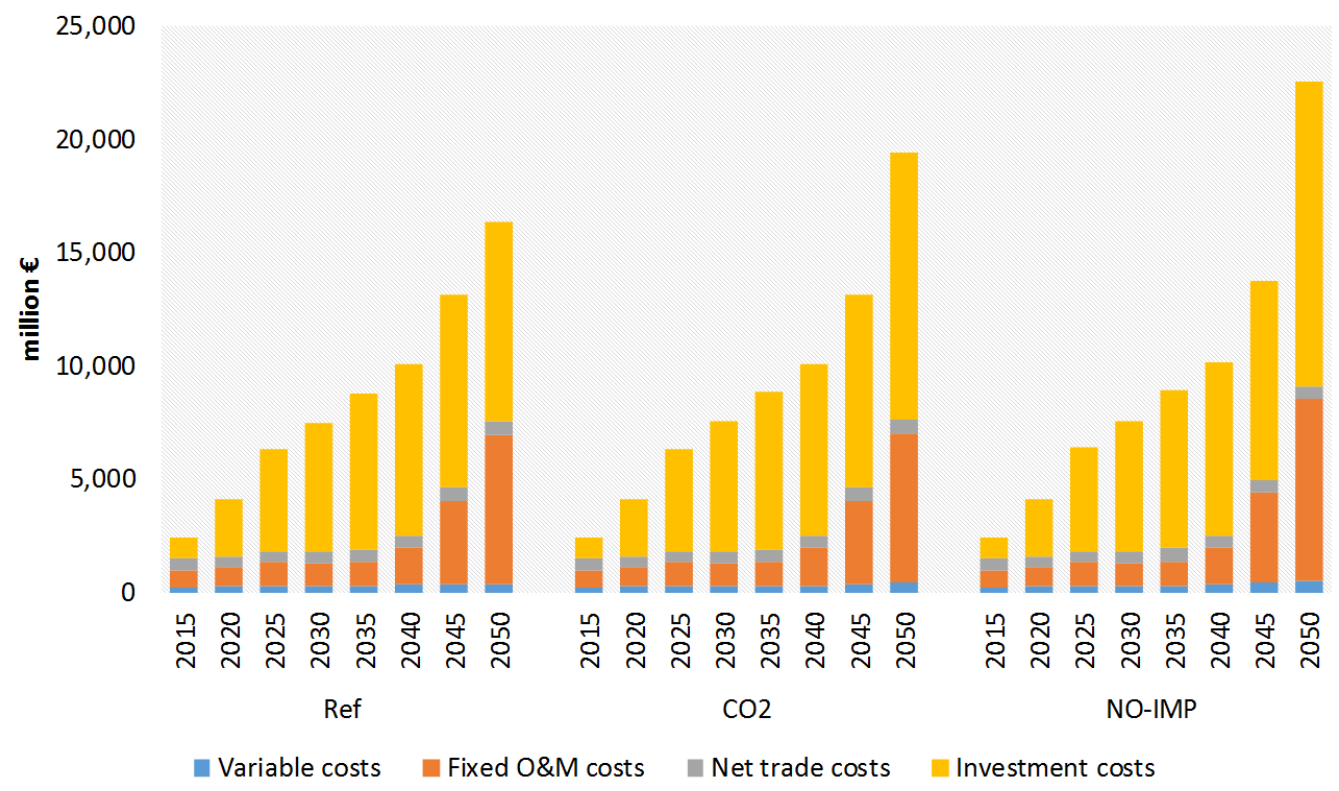

Figure 17: Annual system costs by type in the analysed scenarios (sunk costs are not included).

demand) in the CO2, and $926 \mathrm{GWh}$ (3\% of the total DH demand) in the NO-IMP.

Incineration $\mathrm{WtE}$ plants can cover up to $25 \%$ of the $\mathrm{DH}$ demand in central areas, while industrial excess heat can supply around 1\%. Incineration plants are centralised due to the meaningful impacts from economy of scale, as discussed by Pizarro-Alonso, Cimpan, Ljunggren Söderman, Ravn, and Münster (2018a). They are located in the areas with the largest populations, hence the highest waste generation and minimum transport costs, and large district heating demands.

Biorefineries need to be relatively centralised due to the impacts from economies of scale, with the possibility to install up to five straw biorefineries in Denmark, if the full potential is utilised. Comparing Figures 9 and 18, the optimal location of straw biorefineries is largely driven by the possibility to use the excess heat, hence they will be located in areas with relatively large district heating demands (red areas in Figure 3 (a)), and their location will also depend on the need to minimise transport of resources, but subject to the requirements from economies of scale. In the CO2 scenario less than $11 \%$ of the heat produced by biorefineries would have to be cooled down by 2050.

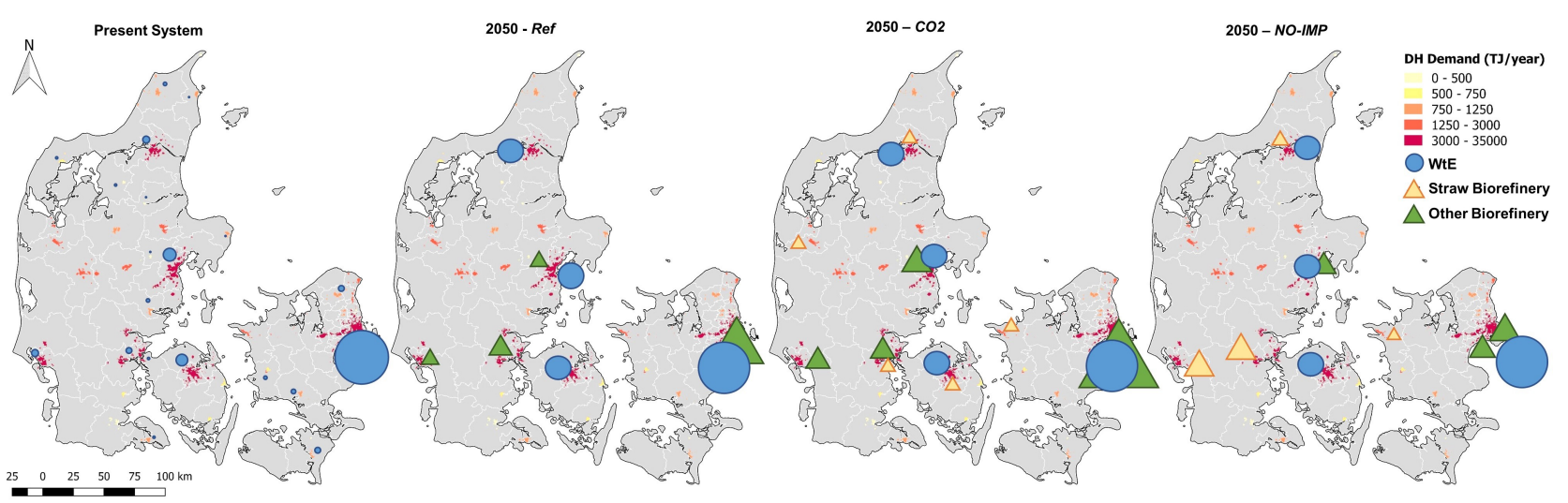

Figure 18: Location of waste incineration plants and biorefineries as a result from Balmorel-OptiFlow (the size of the symbols is proportional to the amount of district heating provided).

Figure 18 shows that there is a coexistence of different biorefineries and incineration plants within the largest district heating networks. Nevertheless, if the possibility to install inter-seasonal heat 
storage is limited, location of incineration plants remains similar, while biorefineries would be placed in smaller district heating networks. In this way, the competition for supplying the base-load heating demand would be minimised, as well as the cooling down of heat, despite the fact that it would increase to approximately $20 \%$.

In addition to the straw-based biorefineries, in the Reference and CO2 scenarios, four biorefineries for biodiesel production from woodchips/wood pellets are built in 2050, and located in the largest $\mathrm{DH}$ grids to efficiently recover the large amount of excess heat provided. Similarly, in the NO-IMP scenario, two biorefineries for biodiesel production and one for methanol production from domestic woodchips are constructed, also located in the largest DH grids. Other biorefineries, such as biodiesel from rapeseed and bioethanol from corn in the Reference and NO-IMP scenarios, have very low excess heat production, and their optimal location is driven by the location of their feedstocks, being preferentially placed in smaller DH grids.

\subsection{Greenhouse gas emissions assessment}

The decrease in fossil emissions from 2015 to 2050 shown in Figure 19 is explained by a transition away from fossil resources, especially in the power and district heating sectors, with increasing investments in wind, solar power and heat pumps, along with the introduction of electric vehicles and biofuels in the transport sector. Given the current decrease in costs for renewable technologies and demand electrification, and the economic benefits associated to the implementation of moderate energy efficiency measures, the Reference scenario already experiences a deep decrease in fossil emissions without specific climate policies. The remaining fossil emissions in 2050 for the Reference scenario come from industry, heavy transport and waste incineration plants. In both the CO2 and the NO-IMP scenarios, fossil $\mathrm{CO}_{2}$ emissions are almost fully abated by 2050 , through an increased use of renewable-based electricity and bioenergy in industry, freight, aviation and shipping, as well as an increase in the energy efficiency.

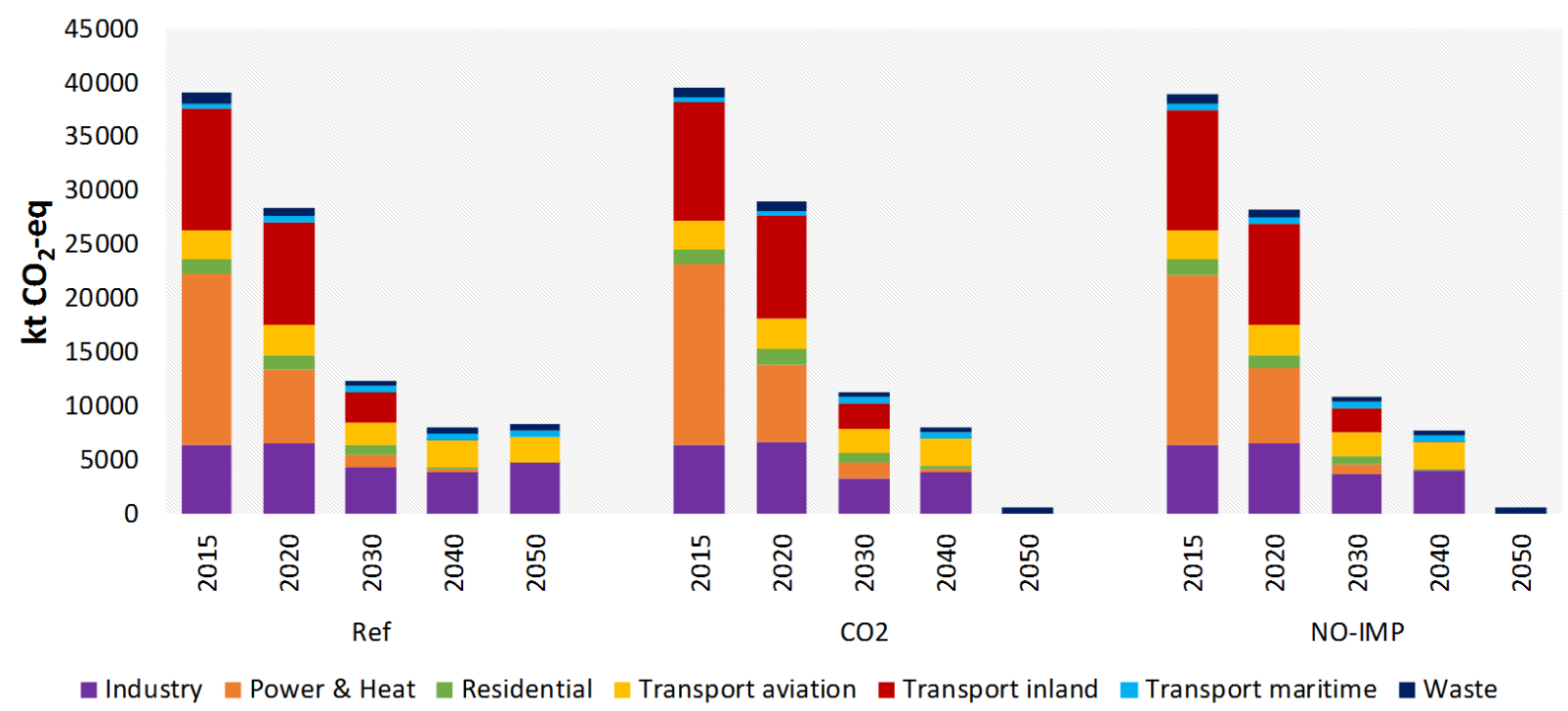

Figure 19: Evolution of fossil $\mathrm{CO}_{2}$ emissions from the energy system in the analysed scenarios.

The evolution of biogenic emissions, as reported in Figure 20, shows that emissions increasing the net balance are related to the ILUC effects associated with first-generation biomass, emissions associated to indoor and outdoor storage of raw manure, and fugitive losses of methane from biogas production in the anaerobic digesters. On the contrary, negative biogenic emissions from bioashes and spread of digestate on land contribute to restoring the soil carbon balance.

In the Reference, the net positive biogenic emission balance is explained by the ILUC effects from woodchips being larger than the benefits provided by applying the bioashes from gasification on soil. In both the CO2 and the NO-IMP scenarios, negative biogenic emissions increase in the future, 


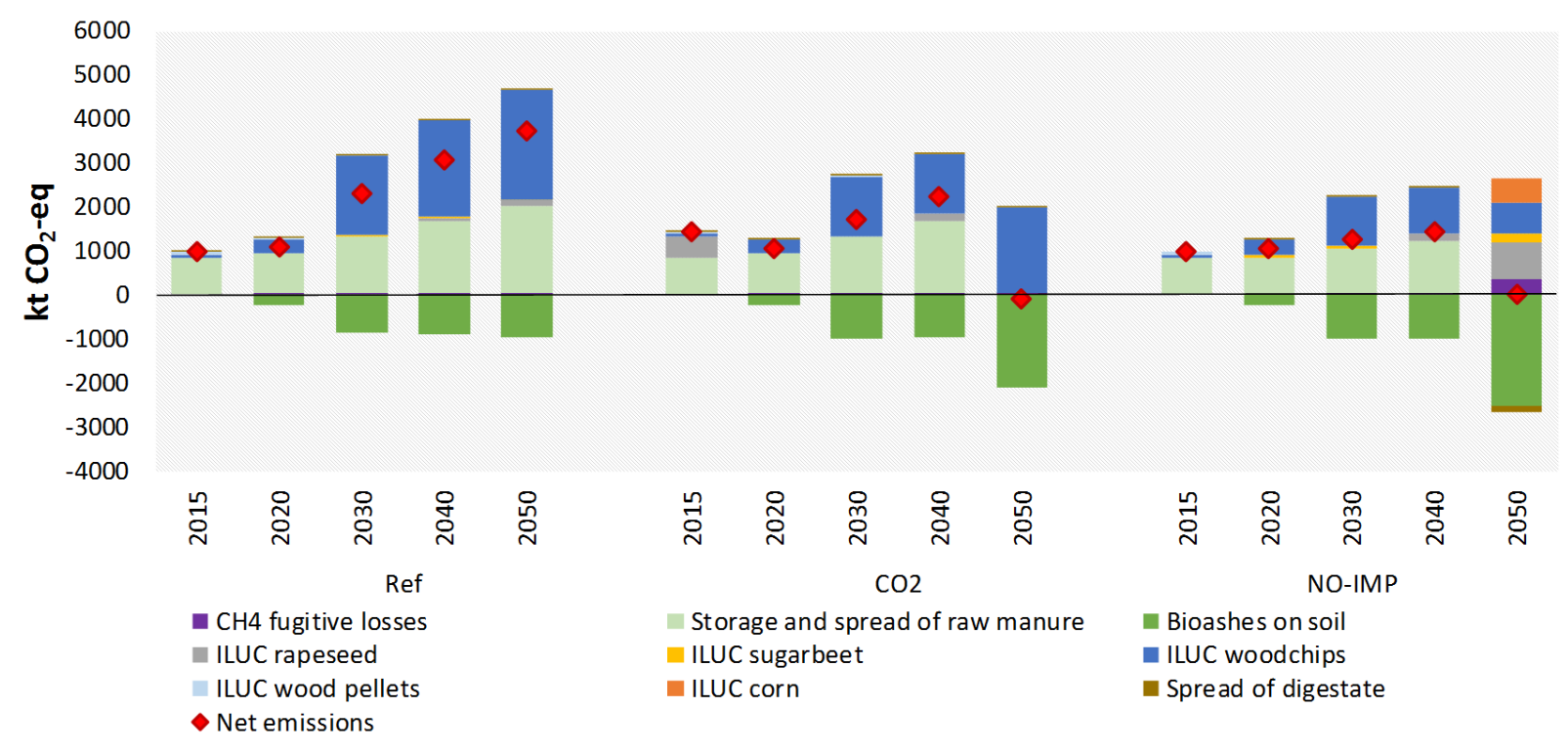

Figure 20: Evolution of biogenic $\mathrm{CO}_{2}$-eq emissions from the energy system in the analysed scenarios including ILUC.

following from the increased gasification of woody biomass and straw for the production of biofuels, thus the subsequent application of bioashes on land.

In the Reference, $25 \%$ of the manure available for anaerobic digestion is cost-effectively diverted to produce biogas, while $75 \%$ is still spread on land by 2050. Emissions from spread of raw manure on land increase over time, as it is assumed that the manure potential available for anaerobic digestion increases (Table 2). In the CO2 and NO-IMP scenarios, all manure available for energy is destined to anaerobic digestion by 2050 , due to the need to minimise emissions from raw spread on-land. Furthermore, avoided emissions from the application of digestate from manure co-digestion with the organic fraction of waste and induced emissions from digestate from manure mono-digestion are present in the three scenarios. However, their impact on the biogenic carbon balance is very limited. In the NO-IMP scenario, negative emissions associated to the spreading of digestate from anaerobic co-digestion of manure with grass and sugarbeet are also present, as illustrated in Figure 5.

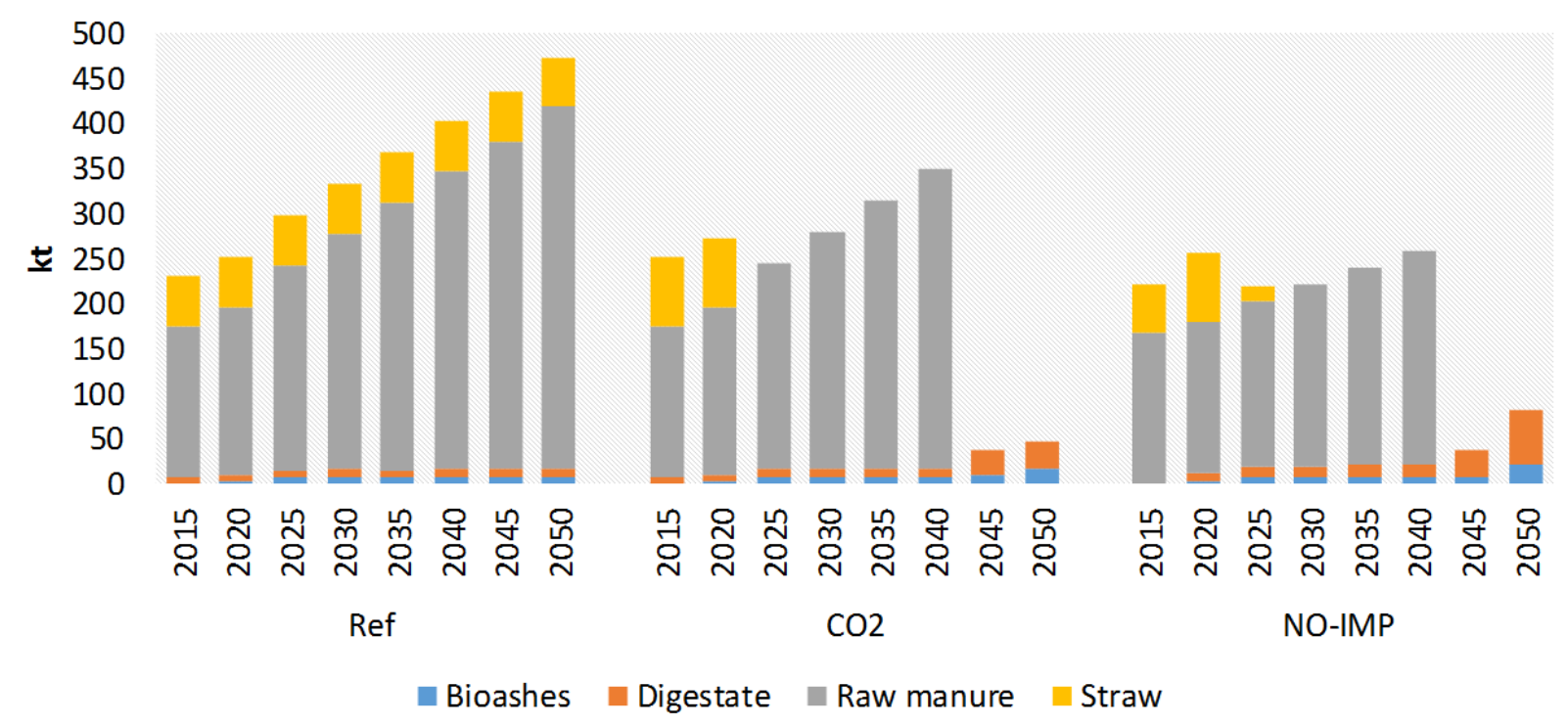

Figure 21: Avoided fertilisers $(\mathrm{N}, \mathrm{P}, \mathrm{K})$ by substituting source in the analysed scenarios.

TIMES-DK accounts for the avoided fertiliser production achieved through substituting soil fertilising 
effects through ploughing straw on field, spreading raw manure, recovering and spreading on land the digestate from anaerobic digestion and bioashes from gasification processes (Section 2.4). Figure 21 provides an account of the fertilising effects achieved by the different processes across the three analysed scenarios. While fertilisers from spreading raw manure are dominant and increasing over the years in the Reference scenario, these are sharply disappearing in the last years of the modelling horizon (2045-2050) under more constrained scenarios (CO2 and NO-IMP), as a result of diverting animal manure to the more climate-effective anaerobic digestion plants. Digestate recovered from these plants can thereafter be spread on land for soil fertilising effects, increasing especially in the NO-IMP scenario where more biogas plants are invested in.

\subsection{Sensitivity analysis}

Biomass might represent a large share of the Danish energy consumption in the future, both under business as usual conditions, but especially in a stringent climate mitigation scenario. Biomass will likely play a key role in the supply of fuels to industry, heavy-duty transport and aviation sectors. From this point of view, the availability of sustainable domestic biomass is crucial to minimise the environmental impacts of the future energy system. The domestic potential of first-generation biomass destined to the energy sector, e.g. sugarbeet and rapeseed, is uncertain, and it depends on factors such as land use, land availability, climate conditions and economic feasibility of fuel conversion pathways.

Figure 22 (left) illustrates the impact of the availability of Danish energy crops on the degree of energy self-sufficiency for the NO-IMP scenario in 2050. Bioenergy imports in the CO2 scenario amount to $73 \mathrm{PJ}$ of woodchips and $48 \mathrm{PJ}$ of biofuels by 2050. In the NO-IMP scenario, a larger availability of rapeseed and sugarbeet, primarily destined to biogas and biodiesel production, diminishes the need for imports of biokerosene from abroad, albeit with the same impact on the use of straw, both under a $50 \%$ and a $100 \%$ increase. This effect can be explained by the fact that all the available straw is already fully utilised for the production of biokerosene in the $+50 \%$ case. On the other hand, a lower potential of energy crops increases the need for imports of aviation fuel, unless alternative production routes for biokerosene become commercially feasible. The potential of straw available for energy is currently uncertain, and a further analysis could consider soil-specific rates of straw removal, while maintaining safe and sustainable levels of soil organic carbon (SOC), structure and water retention (Blanco-Canqui and Lal, 2009). By analogy with these modelling results, a lower availability of straw would increase biokerosene imports.
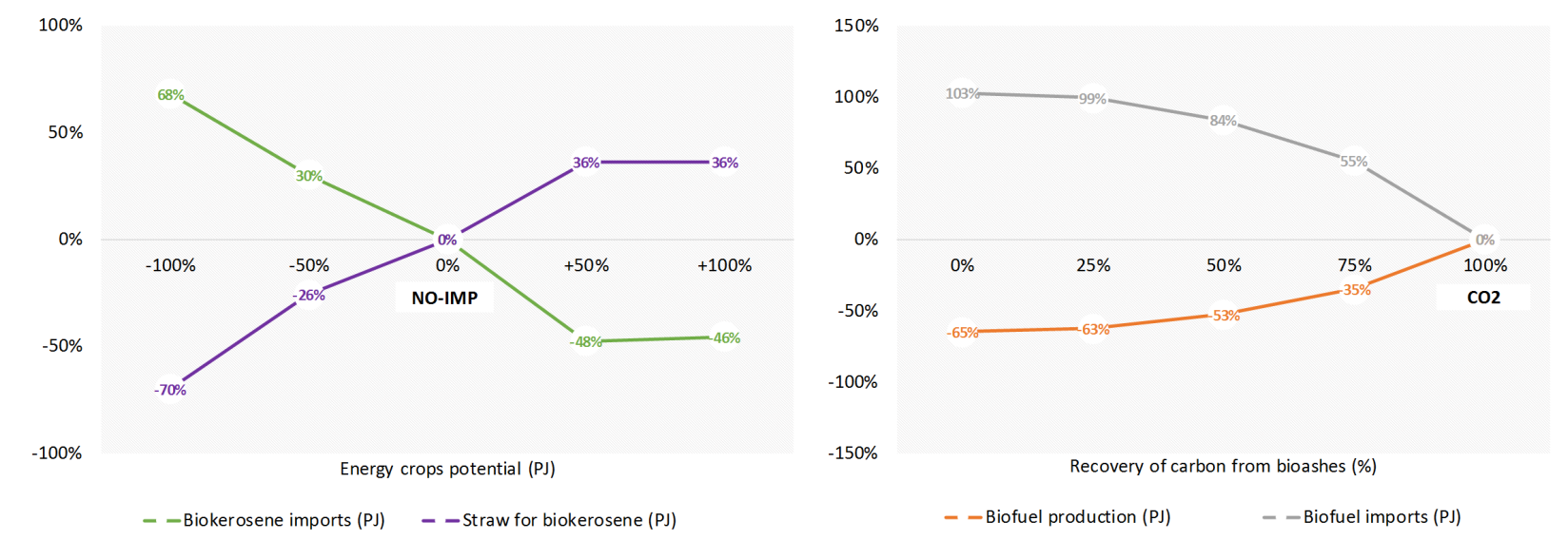

Figure 22: Impact of energy crops availability on the domestic biofuel supply and straw utilisation - Sensitivity analysis for the NO-IMP scenario in 2050 (left) \& Impact of carbon recovery from recycling of bioashes on biofuel production and imports - Sensitivity analysis for the CO2 scenario in 2050 (right).

In the perspective of adopting a more circular use of domestic resources, the recycling of the byproducts from biomass gasification back into agricultural fields can enhance soil nutrients and organic carbon. The application of biochar on Danish soil demonstrated the potential of substituting 
mineral fertiliser and traditional lime (Hansen, Muller-Stover, Imparato, Krogh, Jensen, Dolmer, and Hauggaard-Nielsen, 2017), while it presented no negative effects on soil microorganisms and earthworms. Moreover, biochar in sandy soils has a positive influence on water holding capacity and soil structure (Ahrenfeldt, Thomsen, Henriksen, and Clausen, 2013). However, the contribution to restoring the SOC is more debated and location-dependent, with some studies showing no effects on SOC from biochar application on soil (Hansen, Muller-Stover, Imparato, Krogh, Jensen, Dolmer, and Hauggaard-Nielsen, 2017), and other analyses concluding on the positive climate impact of carbon soil sequestration through biochar produced from straw gasification (Sigurjonsson, Elmegaard, Clausen, and Ahrenfeldt, 2015).

In this view, we performed a sensitivity analysis on the CO2 scenario, testing decreasing levels of carbon recovering from bioashes of biomass gasification, with $100 \%$ corresponding to the base level, as illustrated in Figure 22 (right). Decreasing levels of carbon recovery from application on soil result in reduced domestic biofuel production and corresponding increased reliance on biofuel imports, especially second-generation biodiesel. This trend can be explained by the progressive diminishing benefit of using bioashes as a carbon sink, which contributes to the climate neutrality goal, thus opting for cheaper biofuels produced abroad (for which sustainability needs to be ensured).

\section{Discussion}

\subsection{Technology prioritisation}

Residual biomass resources become increasingly valuable for the production of sustainable fuels and heat, particularly under stringent climate mitigation scenarios and energy self-sufficiency goals. Under these conditions, a socio-economic optimization prioritises the gasification route with subsequent Fischer-Tropsch synthesis to biofuels, i.e. biodiesel and biokerosene, to supply the heavy segments of the transport sector. Additionally, the possibility of recovering the excess heat from biorefinery plants in central DH networks, as well as recycling carbon and nutrients into the soil, contributes to the attractiveness of this route. On the other hand, although first-generation biomass is domestically available, indirect land-use change effects hinder its utilisation for the production of biofuels or biogas in a carbon-constrained scenario.

While biomass gasification technologies coupled with FT synthesis represent a promising option for the production of alternative transport fuels, further technology development and scaling are needed to achieve commercial installations (Sikarwar, Zhao, Clough, Yao, Zhong, Memon, Shah, Anthony, and Fennell, 2016). However, large-scale applications exists for the biomass gasification technology in isolation, e.g. for the cogeneration of heat and electricity (Ahrenfeldt, Thomsen, Henriksen, and Clausen, 2013). Among possible technological improvements, gas yields can be further optimized by increasing gasification temperatures (Qin, Lin, Jensen, and Jensen, 2012), and by pre-heating and drying the biomass (Alamia, Larsson, Breitholtz, and Thunman, 2017), while the production of tar and soot can be limited by adjusting the injection of steam and excess air in the process (Qin, Lin, Jensen, and Jensen, 2012).

The comparison with other energy system assessments in the Danish context reveals relatively similar results. The possibility of boosting biofuel production from thermal gasification through syngas hydrogenation has been highlighted also in other studies (Connolly, Mathiesen, and Ridjan, 2014), representing a valuable option, particularly when assuming limited availability of biomass. In line with the results of the NO-IMP scenario, domestic biomass resources are in the long run insufficient to supply the total Danish fuel demand (Tonini, Vadenbo, and Astrup, 2017), (Larsen, Jepsen, and Frederiksen, 2013), unless adopting different cropping systems, improving harvest practices and increasing land dedicated to fast-growing forest biomass (Gylling, Jørgensen, Bentsen, Kristensen, Dalgaard, Felby, Larsen, and Johannsen, 2016).

Other studies conducting pure environmental optimization of technology pathways for residual biomass revealed the combustion of straw for cogeneration of heat and power as the preferred alternative (Tonini, Vadenbo, and Astrup, 2017), as it displaces coal- and gas-fired heat and power generation, with a temporal scope up to 2030; while manure-straw co-digestion for biogas production is preferred 
over second generation bioethanol in Wenzel, Høibye, Duban Grandal, Hamelin, Bird, and Olesen (2014), with a time frame up to 2050. However, in Tonini, Vadenbo, and Astrup (2017), when imposing renewable targets on transport fuels and enforcing a phase-out of coal and oil, part of the straw resources are diverted to bioethanol production, and part of the woody biomass to thermal gasification and FT synthesis. The divergent technology optimization arises from different modelling horizons, conversion pathways and dimensions covered in the studies, i.e. the economic dimension was not included in neither Tonini, Vadenbo, and Astrup (2017) nor Wenzel, Høibye, Duban Grandal, Hamelin, Bird, and Olesen (2014). Moreover, in Wenzel, Høibye, Duban Grandal, Hamelin, Bird, and Olesen (2014) it was assumed that no carbon goes back to the soil from gasification processes. Our analysis adopts a long-term vision up to 2050, when Denmark aims to be fossil-independent and climate-neutral. In this perspective, decarbonisation of heavy transport and high-temperature process heating are also required. Cogeneration or bioethanol routes could result in lock-in pathways with the possibility to generate stranded assets if a full decarbonisation is pursued in the Danish energy system, thus highlighting the importance of using adequate foresight horizons when assessing long-term goals in the short- and medium-term energy planning.

Regarding the decarbonization of the industrial sectors, we have allowed a complete fuel switch from coal in the Reference scenario towards other sources (gas, biogas, synthetic natural gas, electricity and biomass) in the CO2 and NO-IMP scenarios. Although this fuel substitution might be feasible for many of the industrial sectors operating in Denmark currently and in the foreseeable future, it might be more challenging for the heavy segments, i.e. glass, concrete and metal industries, where radical changes would be required in the industrial processes and/or in the industry configuration in Denmark.

\subsection{Modelling approach}

This study analysed the optimal use of straw from a socio-economic perspective, considering the competing allocation options in different end-use sectors by applying the energy system optimization model TIMES-DK. A holistic multi-sectoral approach is required to represent a more integrated system that allows to achieve a high degree of decarbonisation in a cost-efficient way. On the other hand, this study has not considered the demand of biomass resources for materials, which might become increasingly important, e.g. given the fact that the European Parliament approved on October 2018 a ban on single-use plastics by 2021, due to rising concerns on plastic pollution in the ocean. Therefore, further analyses could be extended to integrate the demand for materials, which might compete with the energy sectors for biomass resources, among others.

This study introduced a novel model linkage between TIMES-DK and the detailed spatio-temporal optimization model Balmorel-OptiFlow, with the latter able to capture transport of resources, dispatch dynamics and chronologies. Furthermore, it includes endogenous electricity trade between Denmark and four neighbouring countries (Sweden, Norway, Germany and Finland), the representation of the 34 largest district heating networks in Denmark as unique entities without aggregation, and the consideration of economies of scale for biorefineries and waste-to-energy plants by introducing binary variables. This modelling framework is computationally demanding due to the need to use MILP, as well as a large number of time slices and geographical divisions for achieving a disaggregated representation of $\mathrm{DH}$ and electricity trade dynamics.

As a large part of the excess heat from biorefineries can be efficiently used in Danish DH networks by optimizing heat storage, the linkage of TIMES-DK with Balmorel-OptiFlow does not modify the conclusions regarding the optimal use of straw. Nevertheless, the use of the Balmorel-OptiFlow model allows not only to show the optimal use of straw, but also the techno-economical feasibility of investments in biorefineries. Results indicate specific information regarding how many biorefineries might be needed, where they could be located or heat storage requirements, all of which can provide relevant support to decision-making in energy planning and, in a broader perspective, public policy. Therefore, depending on the specific questions to be addressed, the use of TIMES-DK as a standalone model might be sufficient to provide more aggregated information on the energy system, e.g. the optimal use of straw. Nevertheless, to determine the actual feasibility of these results (e.g. as infor- 
mation to the public debate), the application of models with detailed spatial and temporal resolution is recommended despite the computational drawbacks.

\subsection{Policy implications}

In Denmark, the existence of large-scale energy producers has been instrumental in developing the straw to energy market (Bentsen, Nilsson, and Larsen, 2018), mainly through the refurbishment of coal-fired power plants. However, in compliance with stringent climate targets, residual biomass should be more effectively diverted to the production of transportation fuels, as to supply the heavyduty segments where the use of electricity presents a challenge.

In contrast with the modelling results, overnight investments in biorefinery technologies, as well as connections with DH networks, may however not be immediately technically feasible in reality. These would instead require the development of efficient supply chains, the formulation of new business models (e.g. recycling of by-products and farmers compensation), the advancement in technology R\&D (e.g. thermal gasification of straw linked to production of liquid fuels) and, not least, financing and political support. Currently, the value chains supporting the combustion of straw for power and heat production in boilers and CHP plants present a variety of formal contracts and informal agreements among the actors involved, according to the size of the plant (Bentsen, Nilsson, and Larsen, 2018). These include the operations from straw harvest to final disposal of ashes. The success of straw-to-biofuel applications would entail a more regulated commodity pricing coupled with sustainability labelling of first-generation biomass for energy use, as well as formalised and operative climate targets or carbon pricing, especially targeting the transport sector.

This study focused on Denmark, where straw is mostly left-on-field, or used for fodder, bedding or energy. However, in many parts of the world, peasants often burn the straw in-situ after the harvest, in spite of the fact that local governments usually ban this practice (Lohan, Jat, Yadav, Sidhu, Jat, Choudhary, Peter, and Sharma, 2018), (Chen and Zhang, 2015), (Forster-Carneiro, Berni, Dorileo, and Rostagno, 2013). Farmers need to remove straw fast to plant crops for the next growing season, with straw burning being a cheap and immediate solution (Zhang, Liu, and Hao, 2016). Straw burning damages the air quality, and subsequently health, and visibility and mobility due to the creation of haze and smog (Li, Li, Li, Tian, Zhao, Wang, Wang, and Shi, 2016), (Zhang, Liu, and Hao, 2016), (Kumar and Kumar, 2016). There are also climate effects due to the emissions of black carbon (Chen, Li, Ristovski, Milic, Gu, Islam, Wang, Hao, Zhang, He, Guo, Fu, Miljevic, Morawska, Thai, LAM, Pereira, Ding, Huang, and Dumka, 2017), and a decrease in the soil quality. Furthermore, straw burning might lead to uncontrollable fires (San-Miguel-Ayanz, Schulte, Schmuck, and Camia, 2013), which can threaten people's lives, infrastructures and ecosystems. The persistent challenges related to deposition of agricultural residues require integrated policies from the agricultural and energy spheres to divert straw towards energy production or biorefineries if deemed optimal, while preserving soil organic carbon stocks (Chen and Zhang, 2015). The use of integrated energy systems analysis models, including the spatio-temporal availability of straw, as shown in this study, could support the decision-making processes and policy definition.

\section{Conclusions}

This study investigated alternative pathways for the optimal use of straw in the Danish context, applying both techno-economic and environmental considerations, under a range of climate and resource-constrained scenarios. The optimal allocation of straw was assessed with the bottom-up optimization model TIMES-DK. Afterwards, the techno-economical feasibility of straw biorefineries, as well as their optimal location and size, was analysed through the use of the Balmorel-OptiFlow model, having a detailed representation of geography and time. The modelling approach showed that a more aggregated representation of spatial and temporal dimensions might be sufficient to assess the optimal use of straw. Nevertheless, a detailed modelling might still be valuable to determine its techno-economic feasibility under a systems perspective. 
The performed socio-economic optimization revealed the increased value of straw in a future decarbonised energy system, especially when energy self-sufficiency targets are pursued, e.g. due to security issues or concerns about the origin, thus the sustainability, of foreign biomass feedstocks. Modelling results highlighted the attractiveness of the gasification route with subsequent Fischer-Tropsch synthesis for the production of biofuels, i.e. biodiesel and biokerosene, to supply the heavy segments of the transport sector under carbon-constrained scenarios. In addition, a holistic planning of biorefineries, electricity and district heating systems increases the utilisation of excess heat recovery from biorefineries, which further maximises the value of straw and minimises the total cost of satisfying end-use demands.

The utilisation of straw for heating or cogeneration in future carbon-constrained scenarios is limited, due to the higher value associated to its use in sectors where electrification and decarbonisation are complex. Nevertheless, in the Reference scenario, straw is a valuable resource for electricity, but mostly for district heating production, as straw boilers can balance the production from other sources, such as heat pumps, and winter peaks; moreover, electricity generation by wind, balanced with electricity trade, is very cost-competitive. Biogas production does not represent a cost-optimal alternative for straw, as its high lignin content would require an expensive pre-treatment process. Finally, straw is not used for bioethanol production through fermentation, as bioethanol is mostly used in light-duty vehicles, which can be electrified.

Modelling results indicated that relying on domestic biomass resources would not attain energy selfsufficiency in a carbon-constrained case, if assuming no drastic changes in diet, crop production and land management practices. Nevertheless, if hydrogen, electrofuels or ammonia, among other technological innovations, become commercially feasible alternatives for the supply of liquid and gaseous fuels, biomass demand would decrease. Among these options, direct air carbon capture could provide the needed $\mathrm{CO}_{2}$ feedstock, which can be combined with hydrogen to produce carbon-neutral fuels (Blanco, Nijs, Ruf, and Faaij, 2018). Therefore, further research should advance technology pathways for straw utilisation and the development of an operative regulatory framework, as well as advance innovative solutions for those sectors that cannot be electrified. This would promote the transition towards climate neutrality in a cost-efficient way, while both pursuing the target of energy self-sufficiency and minimising the need for biomass, as its sustainability remains uncertain.

It is challenging to achieve carbon neutrality unless carbon sinks increase, such as natural carbon sinks or artificial techniques for carbon sequestration. Straw use might bring net negative impacts on the Danish greenhouse gases balance when applying bioashes from gasification for restoring soil nutrients and carbon in agriculture fields. However, these impacts are still uncertain. Emissions associated to indirect land-use changes from other biomass feedstocks should still be compensated with an increase in carbon sinks to achieve the desired goal of climate neutrality, even more if the global demand for biomass rises and land governance is imperfect, thus resulting in higher indirect emissions to be offset.

\section{Acknowledgements}

The work reported in this article has been undertaken as part of the COMETS project, financed by the Innovation Fund Denmark (grant number 4106-00033B).

\section{Appendix}

The following Supplementary Material provides the background data and assumptions used in this article:

- Supplementary Material A: Technology data for biorefineries and biogas plants.

- Supplementary Material B: Straw generation in Denmark.

- Supplementary Material C: Exogenous assumptions for socio-economic scenarios. 


\section{References}

AgroTech. Biomass for biogas plants in Denmark in the short and long term (In Danish), 2013. 13

J. Ahrenfeldt, T. Thomsen, U. B. Henriksen, and L. R. Clausen. Biomass gasification cogeneration A review of state of the art technology and near future perspectives. Applied Thermal Engineering, 50(2):1407-1417, 2013. ISSN 18735606, 13594311. doi: 10.1016/j.applthermaleng.2011.12.040. 26

A. Alamia, A. Larsson, C. Breitholtz, and H. Thunman. Performance of large-scale biomass gasifiers in a biorefinery, a state-of-the-art reference. International Journal of Energy Research, 41(14): 2001-2019, 2017. ISSN 1099114x, 0363907x. doi: 10.1002/er.3758. 26

F. M. Andersen and H. V. Larsen. FRIDA: A model for the generation and handling of solid waste in Denmark. Resources, Conservation and Recycling, 65(Supplement C):47 - 56, 2012. doi: 10.1016/j.resconrec.2012.04.004. 13, 14

T. Astrup, D. Tonini, L. Hamelin, and H. Wenzel. Environmental assessment of renewable energy scenarios towards 2050. Technical report, Department of Development and Planning, Aalborg University, 2011. 2

O. Balyk, K. S. Andersen, S. Dockweiler, M. Gargiulo, K. Karlsson, R. Næraa, S. Petrović, J. Tattini, L. B. Termansen, and G. Venturini. Times-dk: Technology-rich multi-sectoral optimisation model of the danish energy system. Energy Strategy Reviews, 23:13-22, 2019. doi: 10.1016/j.esr.2018.11. 003. $4,6,11$

N. S. Bentsen, D. Nilsson, and S. Larsen. Agricultural residues for energy - A case study on the influence of resource availability, economy and policy on the use of straw for energy in Denmark and Sweden. Biomass and Bioenergy, 108:278-288, 2018. ISSN 18732909, 09619534. doi: 10.1016/ j.biombioe.2017.11.015. 2, 21, 28

G. Berndes, M. Hoogwijk, and R. Van Den Broek. The contribution of biomass in the future global energy supply: A review of 17 studies. Biomass and Bioenergy, 25(1):1-28, 2003. ISSN 09619534. doi: 10.1016/S0961-9534(02)00185-X. 1

H. Blanco, W. Nijs, J. Ruf, and A. Faaij. Potential for hydrogen and Power-to-Liquid in a lowcarbon EU energy system using cost optimization. Applied Energy, 232:617 - 639, 2018. doi: 10.1016/j.apenergy.2018.09.216. 29

H. Blanco-Canqui and R. Lal. Crop Residue Removal Impacts on Soil Productivity and Environmental Quality. Critical Reviews in Plant Sciences, 28(3):139-163, 2009. doi: 10.1080/ 07352680902776507. 14, 25

A. Boldrin, K. R. Baral, T. Fitamo, A. H. Vazifehkhoran, I. G. Jensen, I. Kjærgaard, K.-A. Lyng, Q. van Nguyen, L. S. Nielsen, and J. M. Triolo. Optimised biogas production from the co-digestion of sugar beet with pig slurry: Integrating energy, GHG and economic accounting. Energy, 112: 606-617, 2016. doi: 10.1016/j.energy.2016.06.068. 11, 12

A. Brosowski, D. Thraen, U. Mantau, B. Mahro, G. Erdmann, P. Adler, W. Stinner, G. Reinhold, T. Hering, and C. Blanke. A review of biomass potential and current utilisation - Status quo for 93 biogenic wastes and residues in Germany. Biomass and Bioenergy, 95:257-272, 2016. ISSN 18732909, 09619534. doi: 10.1016/j.biombioe.2016.10.017. 1

S. Brynolf, M. Taljegard, M. Grahn, and J. Hansson. Electrofuels for the transport sector: A review of production costs. Renewable and Sustainable Energy Reviews, 81:1887-1905, 2018. ISSN 18790690, 13640321. doi: 10.1016/j.rser.2017.05.288. 3 
F. Bühler, T.-V. Nguyen, and B. Elmegaard. Energy and Exergy Analyses of the Danish Industry Sector. Applied Energy, 184:1447-1459, 2016. ISSN 18729118, 03062619. doi: 10.1016/j.apenergy. 2016.02.072. 15

F. Bühler, S. Petrovic, K. B. Karlsson, and B. Elmegaard. Industrial excess heat for district heating in Denmark. Applied Energy, 205:991-1001, 2017. ISSN 18729118, 03062619. doi: 10.1016/j. apenergy.2017.08.032. 15

H. G. Chen and Y. H. Zhang. New biorefineries and sustainable agriculture: Increased food, biofuels, and ecosystem security. Renewable and Sustainable Energy Reviews, 47:4146, 117-132, 2015. ISSN 18790690, 13640321. doi: 10.1016/j.rser.2015.02.048. 28

J. Chen, C. Li, Z. Ristovski, A. Milic, Y. Gu, M. S. Islam, S. Wang, J. Hao, H. Zhang, C. He, H. Guo, H. Fu, B. Miljevic, L. Morawska, P. Thai, Y. F. LAM, G. Pereira, A. Ding, X. Huang, and U. C. Dumka. A review of biomass burning: Emissions and impacts on air quality, health and climate in China. Science of the Total Environment, 579:1000-1034, 2017. ISSN 18791026, 00489697. doi: 10.1016/j.scitotenv.2016.11.025. 28

F. Cherubini and S. Ulgiati. Crop residues as raw materials for biorefinery systems - A LCA case study. Applied Energy, 87(1):47-57, 2010. ISSN 18729118, 03062619. doi: 10.1016/j.apenergy. 2009.08.024. 2

D. Connolly, B. V. Mathiesen, and I. Ridjan. A comparison between renewable transport fuels that can supplement or replace biofuels in a 100\% renewable energy system. Energy, 73:110-125, 2014. ISSN 03605442. doi: 10.1016/j.energy.2014.05.104. 2, 3, 26

V. Daioglou, B. Wicke, A. P. C. Faaij, and D. P. Van Vuuren. Competing uses of biomass for energy and chemicals: implications for long-term global $\mathrm{CO}_{2}$ mitigation potential. Global Change Biology Bioenergy, 7(6):1321-1334, 2015. ISSN 17571707, 17571693. doi: 10.1111/gcbb.12228. 3

Danish Agricultural Agency. Agricultural Analyses (In Danish), 2016. URL http:// jordbrugsanalyser.dk/downloadside/index.html. Accessed on 28/01/2018. 13

Danish Energy Agency. Analysis of bioenergy in Denmark (In Danish). Technical report, 2013. 2, 13

Danish Energy Agency. Biogas in Denmark - Status, barriers and perspectives (In Danish). Technical report, 2014. 12, 13

Danish Energy Agency. Analysis of the possibilities for promoting production and use of advanced biofuels in Denmark (In Danish), 2016. 3

Danish Energy Agency. Danish Energy and Climate Outlook 2017. Technical report, 2017a. 2

Danish Energy Agency. Socio-economic calculations for energy prices and emissions (In Danish), 2017b. 10

Danish Ministry of Energy, Utilities and Climate. Together for a greener future. Climate and Air proposal (In Danish), 2018. 16

Danish Ministry of the Environment and Food. Order on the use of waste for agricultural purposes (in danish: Bekendtgørelse om anvendelse af affald til jordbrugsformål), 2018. 14

Ea Energy Analyses. Analysis of biomass prices, 2013. 5

EA Energy Analysis. Use of biogas for electricity and heat production. Technical report, 2014. 5

Ecofys, IIASA and E4tech. The land use change impact of biofuels consumed in the EU, 2015. 9 
A. Ekman, O. Wallberg, E. Joelsson, and P. Borjesson. Possibilities for sustainable biorefineries based on agricultural residues - A case study of potential straw-based ethanol production in Sweden. Applied Energy, 102:299-308, 2013. ISSN 18729118, 03062619. doi: 10.1016/j.apenergy.2012.07. 016. 2

Energinet. Energy Data Service, 2018. URL https://www.energidataservice.dk/en/. 10

ENTSO-E. ENTSO-E Transparency Platform, 2018. URL https://transparency.entsoe.eu/. 10

European Commission. The role of waste-to-energy in the circular economy, 2017. 14

European Commission. Directorate-General for Climate Action. Proposal for a REGULATION OF THE EUROPEAN PARLIAMENT AND OF THE COUNCIL on binding annual greenhouse gas emission reductions by Member States from 2021 to 2030 for a resilient Energy Union and to meet commitments under the Paris Agreement and amending Regulation No 525/2013 of the European Parliament and the Council on a mechanism for monitoring and reporting greenhouse gas emissions and other information relevant to climate change, 2016. EEA relevance. 16

European Parliament. Promotion of the use of energy from renewable sources. European Parliament legislative resolution of 13 November 2018 on the proposal for a directive of the European Parliament and of the Council on the promotion of the use of energy from renewable sources (recast) $(\operatorname{COM}(2016) 0767-\mathrm{C} 8-0500 / 2016-2016 / 0382(\mathrm{COD})), 2018.16$

Eurostat. Waste Statistics, 2018. URL http://ec.europa.eu/eurostat/statistics-explained/ index.php/Waste_statistics. 14

A. Evald, G. Hu, and M. T. Hansen. Technology data for advanced bioenergy fuels. Technical report, Force Technology, Department for Biomass and Waste, 2013. 10

N. Forsell, G. Guerassimoff, D. Athanassiadis, A. Thivolle-Casat, D. Lorne, G. Millet, and E. Assoumou. Sub-national TIMES model for analyzing future regional use of biomass and biofuels in Sweden and France. Renewable Energy, 60:415-426, 2013. ISSN 18790682, 09601481. doi: 10.1016/j.renene.2013.05.015. 3

T. Forster-Carneiro, M. D. Berni, I. L. Dorileo, and M. A. Rostagno. Biorefinery study of availability of agriculture residues and wastes for integrated biorefineries in Brazil. Resources, Conservation and Recycling, 77:78-88, 2013. ISSN 18790658, 09213449. doi: 10.1016/j.resconrec.2013.05.007. 28

M. A. Gonzalez-Salazar, M. Venturini, W.-R. Poganietz, M. Finkenrath, T. Kirsten, H. Acevedo, and P. R. Spina. A general modeling framework to evaluate energy, economy, land-use and GHG emissions nexus for bioenergy exploitation. Applied Energy, 178:223-249, 2016. ISSN 18729118, 03062619. doi: 10.1016/j.apenergy.2016.06.039. 3

J. S. Gregg and S. J. Smith. Global and regional potential for bioenergy from agricultural and forestry residue biomass. Mitigation and Adaptation Strategies for Global Change, 15(3):241-262, Mar 2010. ISSN 1573-1596. doi: 10.1007/s11027-010-9215-4. 1

M. Guo, W. Song, and J. Buhain. Bioenergy and biofuels: History, status, and perspective. Renewable and Sustainable Energy Reviews, 42:712-725, 2015. ISSN 18790690, 13640321. doi: 10.1016/j.rser. 2014.10.013. 1

M. Gylling, U. Jørgensen, N. S. Bentsen, I. T. Kristensen, T. Dalgaard, C. Felby, S. Larsen, and V. K. Johannsen. The +10 million tonnes study. Technical report, Department of Food and Resource Economics, University of Copenhagen, 2016. 2, 26

M. B. Hagberg, K. Pettersson, and E. O. Ahlgren. Bioenergy futures in Sweden - Modeling integration scenarios for biofuel production. Energy, 109:1026-1039, 2016. ISSN 18736785, 03605442. doi: 10.1016/j.energy.2016.04.044. 3 
L. Hamelin, U. Jorgensen, B. M. Petersen, J. E. Olesen, and H. Wenzel. Modelling the carbon and nitrogen balances of direct land use changes from energy crops in Denmark: a consequential life cycle inventory. Global Change Biology Bioenergy, 4(6):889-907, 2012. ISSN 17571707, 17571693. doi: 10.1111/j.1757-1707.2012.01174.x. 14

L. Hamelin, I. Naroznova, and H. Wenzel. Environmental consequences of different carbon alternatives for increased manure-based biogas. Applied Energy, 114:774-782, 2014. doi: 10.1016/j. apenergy.2013.09.033. 2, 3, 9, 10, 14

V. Hansen, D. Muller-Stover, V. Imparato, P. H. Krogh, L. S. Jensen, A. Dolmer, and H. HauggaardNielsen. The effects of straw or straw-derived gasification biochar applications on soil quality and crop productivity: A farm case study. Journal of Environmental Management, 186(Part 1):88-95, 2017. ISSN 10958630, 03014797. doi: 10.1016/j.jenvman.2016.10.041. 26

International Energy Agency. Production costs of alternative transportation fuels, 2013. 5

IPCC. IPCC Guidelines for National Greenhouse Gas Inventories, 2006. 9, 16

E. Johnson. Goodbye to carbon neutral: Getting biomass footprints right. Environmental Impact Assessment Review, 29(3):165-168, 2009. doi: 10.1016/j.eiar.2008.11.002. 9

Joint Research Centre. The JRC-EU-TIMES model. Bioenergy potentials for EU and neighbouring countries. Technical report, European Commission, 2015. 11

M. Junginger, T. Bolkesjø, D. Bradley, P. Dolzan, A. Faaij, J. Heinimö, B. Hektor, Øyvind Leistad, E. Ling, M. Perry, E. Piacente, F. Rosillo-Calle, Y. Ryckmans, P.-P. Schouwenberg, B. Solberg, E. Trømborg, A. da Silva Walter, and M. de Wit. Developments in international bioenergy trade. Biomass and Bioenergy, 32(8):717 - 729, 2008. ISSN 0961-9534. doi: 10.1016/j.biombioe.2008.01. 019. 1

S. Kumar and P. Kumar. Economic impact of air pollution from agricultural residue burning on human health. Environmental Science and Engineering (subseries: Environmental Science), (203019): 297-313, 2016. ISSN 18635539, 18635520. doi: 10.1007/978-3-319-31014-5_17. 28

J. K. Kurian, G. R. Nair, A. Hussain, and G. S. V. Raghavan. Feedstocks, logistics and pre-treatment processes for sustainable lignocellulosic biorefineries: A comprehensive review. Renewable and Sustainable Energy Reviews, 25:205-219, 2013. ISSN 18790690, 13640321. doi: 10.1016/j.rser. 2013.04.019. 12

J. Larsen, M. O. Haven, and L. Thirup. Inbicon makes lignocellulosic ethanol a commercial reality. Biomass and Bioenergy, 46(Sp. Iss. SI):36-45, 2012. ISSN 18732909, 09619534. doi: 10.1016/j. biombioe.2012.03.033. 3

L. E. Larsen, M. R. Jepsen, and P. Frederiksen. Scenarios for biofuel demands, biomass production and land use - The case of Denmark. Biomass and Bioenergy, 55:27-40, 2013. ISSN 09619534. doi: $10.1016 /$ j.biombioe.2012.08.015. 2, 26

S. Li, Y. Li, X. Li, X. Tian, A. Zhao, S. Wang, S. Wang, and J. Shi. Effect of straw management on carbon sequestration and grain production in a maize-wheat cropping system in Anthrosol of the Guanzhong Plain. Soil and Tillage Research, 157:43-51, 2016. ISSN 18793444, 01671987. doi: 10.1016/j.still.2015.11.002. 28

S. K. Lohan, H. S. Jat, A. K. Yadav, H. S. Sidhu, M. L. Jat, M. Choudhary, J. K. Peter, and P. C. Sharma. Burning issues of paddy residue management in north-west states of India. Renewable and Sustainable Energy Reviews, 81:693-706, 2018. ISSN 18790690, 13640321. doi: 10.1016/j.rser. 2017.08.057. 28 
R. Loulou, G. Goldstein, A. Kanudia, A. Lettila, and U. Remme. Documentation for the TIMES Model Part I: TIMES Concepts and Theory, 2016. 6

F. Moller, E. Slento, and P. Frederiksen. Integrated well-to-wheel assessment of biofuels combining energy and emission LCA and welfare economic Cost Benefit Analysis. Biomass and Bioenergy, 60:41-49, 2014. ISSN 18732909, 09619534. doi: 10.1016/j.biombioe.2013.11.001. 2

M. Monteleone, A. R. B. Cammerino, P. Garofalo, and M. K. Delivand. Straw-to-soil or strawto-energy? An optimal trade off in a long term sustainability perspective. Applied Energy, 154: 891-899, 2015. ISSN 18729118, 03062619. doi: 10.1016/j.apenergy.2015.04.108. 14

T. L. T. Nguyen, J. E. Hermansen, and L. Mogensen. Environmental performance of crop residues as an energy source for electricity production: The case of wheat straw in Denmark. Applied Energy, 104:633-641, 2013. ISSN 03062619. doi: 10.1016/j.apenergy.2012.11.057. 2

L. S. Nielsen and H. K. Jacobsen. Economies of scale in biogas production and the significance of flexible regulation. Energy Policy, 101:77-89, 2017. ISSN 0301-4215. doi: 10.1016/j.enpol.2016. 11.021. 11

O.-K. Nielsen, M. S. . Plejdrup, M. Winther, M. Nielsen, S. Gyldenkærne, M. H. Mikkelsen, R. Albrektsen, M. Thomsen, K. Hjelgaard, P. Fauser, H. G. . Bruun, V. K. Johannsen, T. Nord-Larsen, L. Vesterdal, I. Callesen, O. H. Caspersen, E. Rasmussen, S. B. Petersen, L. Baunbæk, and M. G. Hansen. Denmark's national inventory report 2017, 2017. 16

E. Panos and R. Kannan. The role of domestic biomass in electricity, heat and grid balancing markets in Switzerland. Energy, 112:1120-1138, 2016. ISSN 18736785, 03605442. doi: 10.1016/j.energy. 2016.06.107. 2

R. Parajuli, T. Dalgaard, U. Jørgensen, A. P. S. Adamsen, M. T. Knudsen, M. Birkved, M. Gylling, and J. K. Schjoerring. Biorefining in the prevailing energy and materials crisis: a review of sustainable pathways for biorefinery value chains and sustainability assessment methodologies. Renewable and Sustainable Energy Reviews, 43:244-263, 2015. ISSN 18790690, 13640321. doi: 10.1016/j.rser.2014.11.041. 1

R. Parajuli, I. S. Kristensen, M. T. Knudsen, L. Mogensen, A. Corona, M. Birkved, N. Peña, M. Graversgaard, and T. Dalgaard. Environmental life cycle assessments of producing maize, grass-clover, ryegrass and winter wheat straw for biorefinery. Journal of Cleaner Production, 142 (4):3859-3871, 2017. ISSN 18791786, 09596526. doi: 10.1016/j.jclepro.2016.10.076. 2

K. Pettersson, E. Wetterlund, D. Athanassiadis, R. Lundmark, C. Ehn, J. Lundgren, and N. Berglin. Integration of next-generation biofuel production in the Swedish forest industry - A geographically explicit approach. Applied Energy, 154:317-332, 2015. ISSN 18729118, 03062619. doi: 10.1016/j. apenergy.2015.04.041. 2, 3

A. Pizarro-Alonso, C. Cimpan, M. Ljunggren Söderman, H. Ravn, and M. Münster. The economic value of imports of combustible waste in systems with high shares of district heating and variable renewable energy. Waste Management, 79:324-338, 2018a. ISSN 18792456, 0956053x. doi: 10. 1016/j.wasman.2018.07.031. 7, 22

A. Pizarro-Alonso, C. Cimpan, and M. Münster. The climate footprint of imports of combustible waste in systems with high shares of district heating and variable renewable energy. Waste Management, 2018b. ISSN 18792456, 0956053x. doi: 10.1016/j.wasman.2018.07.006. 7, 14

D. S. Powlson, A. B. Riche, K. Coleman, N. Glendining, and A. P. Whitmore. Carbon sequestration in European soils through straw incorporation: Limitations and alternatives. Waste Management, 28(4):741-746, 2008. ISSN 18792456, 0956053x. doi: 10.1016/j.wasman.2007.09.024. 2, 14 
K. Qin, W. Lin, P. A. Jensen, and A. D. Jensen. High-temperature entrained flow gasification of biomass. Fuel, 93(1):589-600, 2012. ISSN 18737153, 00162361. doi: 10.1016/j.fuel.2011.10.063. 26

H. Ravn. The Balmorel Model: Theoretical Background. http://balmorel.com/index.php/ balmorel-documentati (Accessed 17 ${ }^{\text {th }}$ March 2019), 2001. 6

H. Ravn. The OptiFlow Model Structure. http://www. balmorel.com/index.php/downloadmodel/ optiflow (Accessed 17 ${ }^{\text {th }}$ March 2019), 2017. 7

C. Robledo-Abad, H. Althaus, G. Berndes, S. Bolwig, E. Corbera, F. Creutzig, J. Garcia-Ulloa, A. Geddes, J. S. Gregg, H. Haberl, S. Hanger, R. Harper, C. Hunsberger, R. K. Larsen, C. Lauk, S. Leitner, J. Lilliestam, H. Lotze-Campen, B. Muys, M. Nordborg, M. Ölund, B. Orlowsky, A. Popp, J. Portugal-Pereira, J. Reinhard, L. Scheiffle, and P. Smith. Bioenergy production and sustainable development: science base for policy-making remains limited. Global Change Biology. Bioenergy, 9(3):541-556, 2017. ISSN 17571707, 17571693. doi: 10.1111/gcbb.12338. 1

S. Samsatli, N. I. J. Samsatli, and N. Shah. BVCM: A comprehensive and flexible toolkit for whole system biomass value chain analysis and optimisation - Mathematical formulation. Applied Energy, 147:131-160, 2015. ISSN 18729118, 03062619. doi: 10.1016/j.apenergy.2015.01.078. 3

J. San-Miguel-Ayanz, E. Schulte, G. Schmuck, and A. Camia. The European Forest Fire Information System in the context of environmental policies of the European Union. Forest Policy and Economics, 29(Sp. Iss. SI):19-25, 2013. ISSN 18727050, 13899341. doi: 10.1016/j.forpol.2011.08.012. 28

N. Scarlat, F. Fahl, J.-F. Dallemand, F. Monforti, and V. Motola. A spatial analysis of biogas potential from manure in Europe. Renewable and Sustainable Energy Reviews, 94:915-930, 2018. ISSN 18790690, 13640321. doi: 10.1016/j.rser.2018.06.035. 2

J. Schmidt, V. Gass, and E. Schmid. Land use changes, greenhouse gas emissions and fossil fuel substitution of biofuels compared to bioelectricity production for electric cars in Austria. Biomass and Bioenergy, 35(9):4060-4074, 2011. ISSN 18732909, 09619534. doi: 10.1016/j.biombioe.2011. 07.007. 2,3

B. Sharma, R. G. Ingalls, C. L. Jones, and A. Khanchi. Biomass supply chain design and analysis: Basis, overview, modeling, challenges, and future. Renewable and Sustainable Energy Reviews, 24: 608-627, 2013. ISSN 18790690, 13640321. doi: 10.1016/j.rser.2013.03.049. 3

K. Shu, U. A. Schneider, and J. Scheffran. Optimizing the bioenergy industry infrastructure: Transportation networks and bioenergy plant locations. Applied Energy, 192:247-261, 2017. ISSN 18729118, 03062619. doi: 10.1016/j.apenergy.2017.01.092. 3

H. Sigurjonsson, B. Elmegaard, L. R. Clausen, and J. Ahrenfeldt. Climate effect of an integrated wheat production and bioenergy system with Low Temperature Circulating Fluidized Bed gasifier. Applied Energy, 160:511 - 520, 2015. doi: 10.1016/j.apenergy.2015.08.114. 2, 9, 10, 26

V. S. Sikarwar, M. Zhao, P. Clough, J. Yao, X. Zhong, M. Z. Memon, N. Shah, E. J. Anthony, and P. S. Fennell. An overview of advances in biomass gasification. Energy and Environmental Science, 9(10):2939-2977, 2016. ISSN 17545706, 17545692. doi: 10.1039/c6ee00935b. 26

M. Spøttle, S. Alberici, G. Toop, D. Peters, L. Gamba, S. Ping, H. van Steen, and D. Bellefleur. Low ILUC potential of wastes and residues for biofuels. Technical report, ECOFYS, 2003. 2

Statistics Denmark. Halm1: Straw yield and use by region, crop, unit and use, 2016. Accessed on 20/01/2018. 2, 13, 16

Statistics Denmark. Bil10: Stock on passenger cars per 1 January by propellant and tare, 2018a. Accessed on 20/01/2018. 5 
Statistics Denmark. Bil15: Stock on vans per 1 January by propellant and gross weight, 2018b. Accessed on 20/01/2018. 5

B. Steubing, R. Zah, and C. Ludwig. Heat, Electricity, or Transportation? The Optimal Use of Residual and Waste Biomass in Europe from an Environmental Perspective. Environmental Science and Technology, 46(1):164-171, 2012. ISSN 15205851, 0013936x. doi: 10.1021/es202154k. 2

The Danish Government. Danish Agreement on Green Growth. Technical report, 2009. 17

The European Parliament and the Council of the European Union. Directive (EU) 2015/1513 of the European Parliament and of the Council of 9 September 2015 amending Directive 98/70/EC relating to the quality of petrol and diesel fuels and amending Directive 2009/28/EC on the promotion of the use of energy from renewable sources, 2015. EEA relevance. 9

D. Tonini, L. Hamelin, and T. F. Astrup. Environmental implications of the use of agro-industrial residues for biorefineries: application of a deterministic model for indirect land-use changes. Global Change Biology. Bioenergy, 8(4):690-706, 2016. ISSN 17571707, 17571693. doi: 10.1111/gcbb. 12290. 2, 3, 13

D. Tonini, C. Vadenbo, and T. F. Astrup. Priority of domestic biomass resources for energy: Importance of national environmental targets in a climate perspective. Energy, 124:295-309, 2017. ISSN 18736785, 03605442. doi: 10.1016/j.energy.2017.02.037. 2, 3, 13, 26, 27

R. Turconi, D. Tonini, C. F. Nielsen, C. G. Simonsen, and T. F. Astrup. Environmental impacts of future low-carbon electricity systems: Detailed life cycle assessment of a Danish case study. Applied Energy, 132:66-73, 2014. ISSN 18729118, 03062619. doi: 10.1016/j.apenergy.2014.06.078. 2

L. van Biert, M. Godjevac, K. Visser, and P. V. Aravind. A review of fuel cell systems for maritime applications. Journal of Power Sources, 327:345-364, 2016. ISSN 18732755, 03787753. doi: 10. 1016/j.jpowsour.2016.07.007. 21

S. van Dyken, B. H. Bakken, and H. I. Skjelbred. Linear mixed-integer models for biomass supply chains with transport, storage and processing. Energy, 35(3):1338-1350, 2010. ISSN 18736785, 03605442. doi: 10.1016/j.energy.2009.11.017. 3

F. Van Stappen, I. Brose, and Y. Schenkel. Direct and indirect land use changes issues in european sustainability initiatives: State-of-the-art, open issues and future developments. Biomass and Bioenergy, 35(12):4824-4834, 2011. ISSN 18732909, 09619534. doi: 10.1016/j.biombioe.2011.07. 015. 9

C. Weiser, V. Zeller, F. Reinicke, B. Wagner, S. Majer, A. Vetter, and D. Thraen. Integrated assessment of sustainable cereal straw potential and different straw-based energy applications in Germany. Applied Energy, 114:749-762, 2014. ISSN 18729118, 03062619. doi: 10.1016/j.apenergy. 2013.07.016. 2

H. Wenzel, L. Høibye, R. Duban Grandal, L. Hamelin, D. N. Bird, and A. Olesen. Carbon footprint of bioenergy pathways for the future Danish energy system. Technical report, 2014. 2, 27

E. Wetterlund, S. Leduc, E. Dotzauer, and G. Kindermann. Optimal localisation of biofuel production on a European scale. Energy, 41(1):462-472, 2012. ISSN 18736785, 03605442. doi: 10.1016/j.energy. 2012.02.051. 3

F. Wiese, R. Bramstoft, H. Koduvere, A. Pizarro Alonso, O. Balyk, J. Kirkerud, Tveten, T. Bolkesjø, M. Münster, and H. Ravn. Balmorel open source energy system model. Energy Strategy Reviews, 20:26 - 34, 2018. doi: 10.1016/j.esr.2018.01.003. 6 
World Bank Group. Commodity Markets Outlook, April. Technical report, 2016. 10

D. Yue, F. You, and S. W. Snyder. Biomass-to-bioenergy and biofuel supply chain optimization: Overview, key issues and challenges. Computers and Chemical Engineering, 66:36-56, 2014. ISSN 18734375, 00981354. doi: 10.1016/j.compchemeng.2013.11.016. 3

L. Zhang, Y. Liu, and L. Hao. Contributions of open crop straw burning emissions to PM2.5 concentrations in China. Environmental Research Letters, 11(1):014014, 2016. ISSN 17489326, 17489318. doi: 10.1088/1748-9326/11/1/014014. 28

J. Zheng and L. Rehmann. Extrusion Pretreatment of Lignocellulosic Biomass: A Review. International Journal of Molecular Sciences, 15(10):18967-18984, 2014. ISSN 14220067, 16616596. doi: 10.3390/ijms151018967. 12 